\title{
Cosmic radio dipole from NVSS and WENSS
}

\author{
M. Rubart and D. J. Schwarz
}

Fakultät für Physik, Universität Bielefeld, Postfach 100131, 33501 Bielefeld, Germany

e-mail: [matthiasr;dschwarz] @physik.uni-bielefeld.de

Received 1 February 2013 / Accepted 30 May 2013

\section{ABSTRACT}

\begin{abstract}
We use linear estimators to determine the magnitude and direction of the cosmic radio dipole from the NRAO VLA Sky Survey (NVSS) and the Westerbork Northern Sky Survey (WENSS). We show that special attention has to be given to the issues of bias due to shot noise, incomplete sky coverage and masking of the Milky Way. We compare several different estimators and show that conflicting claims in the literature can be attributed to the use of different estimators. We find that the NVSS and WENSS estimates of the cosmic radio dipole are consistent with each other and with the direction of the cosmic microwave background (CMB) dipole. We find from the NVSS a dipole amplitude of $(1.8 \pm 0.6) \times 10^{-2}$ in direction $(\mathrm{RA}, \mathrm{dec})=\left(154^{\circ} \pm 19^{\circ},-2^{\circ} \pm 19^{\circ}\right)$. This amplitude exceeds the one expected from the CMB by a factor of about 4 and is inconsistent with the assumption of a pure kinetic origin of the radio dipole at $99.6 \% \mathrm{CL}$
\end{abstract}

Key words. radio continuum: galaxies - large-scale structure of Universe

\section{Introduction}

The assumed isotropy and homogeneity of the Universe at large scales is fundamental to modern cosmology. The isotropy is best seen in the cosmic microwave background (CMB) radiation and holds at the per cent level. The most prominent anisotropy of the CMB temperature is a dipole signal of $\Delta T / T \approx 10^{-3}$. It is commonly assumed that this dipole is largely caused by the motion of the Solar system through the Universe (Stewart \& Sciama 1967). This interpretation seems to be fully consistent with the concordance model of cosmology.

However, the observation of the microwave sky is not enough to tell the difference between a motion induced CMB dipole and dipole contributions form other physical phenomena, i.e.

$\boldsymbol{d}_{\mathrm{cmb}}=\boldsymbol{d}_{\text {motion }}+\boldsymbol{d}_{\text {primordial }}+\boldsymbol{d}_{\mathrm{ISW}}+\boldsymbol{d}_{\text {foregrounds }}+\boldsymbol{d}_{\text {noise }}$.

In our notation a dipole vector $\boldsymbol{d}$ modulates the isotropic sky by a factor $(1+\boldsymbol{d} \cdot \hat{\boldsymbol{r}})$, with $\hat{\boldsymbol{r}}$ denoting the position on the sky.

Usually it is assumed that the primordial and the integrated Sachs-Wolfe (ISW) contribution to the CMB dipole are negligibly small and that foregrounds (the Milky Way) are under control. Within the concordance model we expect a primordial contribution of $d_{\text {primordial }} \approx 2 \times 10^{-5}$. The ISW contribution could be as large as $10^{-4}$ from the gravitational potentials induced by local $100 \mathrm{Mpc}$ sized structures, without being in conflict with the concordance model (Rakic et al. 2006; Francis \& Peacock 2010). The noise term can be ignored due to excellent statistics of full sky observations. Thus the measured $\boldsymbol{d}_{\mathrm{cmb}}$ is directly used to infer the velocity of the Solar system w.r.t. the CMB to be $v=369 \pm 0.9 \mathrm{~km} \mathrm{~s}^{-1}$ (Hinshaw et al. 2009). It is used in many cosmological studies done in the CMB rest frame, e.g. supernova Hubble diagrams or measurements of large scale bulk flows.

The effects of motion are not limited to the CMB, but should actually be detectable at any frequency. In order to test the hypothesis $\boldsymbol{d}_{\mathrm{cmb}}=\boldsymbol{d}_{\text {motion }}$, it would be very interesting to measure the dipole of another cosmic probe, such as that obtained by radio point source catalogues. In this case one expects to find

$\boldsymbol{d}_{\text {radio }}=\boldsymbol{d}_{\text {motion }}+\boldsymbol{d}_{\text {structure }}+\boldsymbol{d}_{\text {foregrounds }}+\boldsymbol{d}_{\text {noise }}$.

Besides the signal from our proper motion, we expect a signal from structure in the Universe and we expect a random dipole from Poisson noise. The dipole from structure is expected to dominate any catalogue limited to redshift $z \ll 1$. Thus we are interested in surveys with a mean redshift of order unity and a large enough sky coverage to be sensitive to the dipole. This makes radio catalogues the preferred probe to look at. Within the concordance model, the dipole signal induced by the large scale structure is then a subdominant contribution, as it is for the CMB. If we had a large enough catalogue, we could compare $\boldsymbol{d}_{\text {radio }}$ to $\boldsymbol{d}_{\mathrm{cmb}}$. Any statistically significant deviation would be exciting, while finding a match would put the concordance model on firmer grounds.

A first attempt to measure the radio dipole was performed by Baleisis et al. (1998) using a combination of the Green Bank 1987 and the Parkes-MIT-NRAO catalogues. Blake \& Wall (2002), Singal (2011) and Gibelyou \& Huterer (2012) attempted to determine the dipole vector in the NRAO VLA Sky Survey (NVSS), with different conclusions. Blake \& Wall (2002) found a result that is in agreement with a purely kinetic origin of the cosmic radio dipole, but this was challenged by Singal (2011), who finds a dipole amplitude four times larger than expected, but strangely enough pointing in a direction consistent with the CMB dipole. The analysis of Gibelyou \& Huterer (2012) finds both a different direction and an amplitude six times as large as the expected one. While Blake \& Wall (2002) used a quadratic estimator, Singal (2011) and Gibelyou \& Huterer (2012) used different linear estimators to find the dipole direction.

The purpose of this work is to discuss the use of linear estimators of the cosmic radio dipole and apply several versions of them on the NVSS (Condon et al. 2002) and the Westerbork 
Northern Sky Survey (WENSS; Rengelink et al. 1997). We resolve the conflicts in the literature and extend the analysis to other linear estimators.

The NVSS survey covers about 10.3 sr of the sky and contains about $2 \times 10^{5}$ sources per steradian. For this survey the Very Large Array (VLA) in New Mexico (USA) has been used measuring at a frequency of $1.4 \mathrm{GHz}$. The survey includes over 80 per cent of the sky, missing only areas with declination $\delta<-40^{\circ}$. The lower flux limit lies at $2.5 \mathrm{mJy}$ for the $5 \sigma$ detection of point sources. The NVSS was conducted by means of two different configurations of the VLA above and below $\delta=-10^{\circ}$.

The Westerbork Synthesis Radio Telescope in the Netherlands was operated at a frequency of $325 \mathrm{MHz}$ to record the WENSS survey covering about 2.9 sr of the nothern sky and containing about $2.3 \times 10^{5}$ sources in total. This survey is made up of a main catalogue for $\delta \in\left(28^{\circ}, 76^{\circ}\right)$ and a polar catalogue for $\delta>72^{\circ}$. The $5 \sigma$ detection limit for this survey is $18 \mathrm{mJy}$.

To analyse these surveys, we focus on linear estimators in this work. We do so for two reasons. Firstly, recent controversial results used linear estimators for the dipole direction (Singal 2011; Gibelyou \& Huterer 2012) and in one work also for the dipole amplitudes (Singal 2011). Secondly, linear estimators are conceptually simpler. However, it is not expected that they are optimal (unbiased and minimal variance). The linear estimators used in our analysis are asymptotically unbiased and their variance can be easily understood by analytic calculations and by Monte Carlo simulations.

The paper is organized as follows: first we discuss the expected kinetic radio dipole. In Sect. 3 we outline previous estimates of the radio dipole. Linear estimators for full sky surveys are investigated in Sect. 4, followed by a detailed analysis of the effects of incomplete sky coverage and masking in the next section. In Sect. 6 we discuss the expected dipole amplitude from a flux based estimator. Our estimate of the radio dipole can be found in Sect. 7 and is followed by a comparison with previous results. We conclude in Sect. 9.

\section{Kinetic radio dipole}

\subsection{Doppler shift and aberration}

Ellis \& Baldwin (1984) predicted the kinetic contribution to the cosmic radio dipole for an isotropic and homogeneous cosmology. At redshift of order unity and beyond, we expect this kinetic contribution to be the dominant one.

The spectrum of a radio source is assumed to be described by a power law,

$S(f) \propto f^{-\alpha}$,

where $S$ denotes the flux and $f$ the frequency. Each radio source can be described by an individual spectral index $\alpha$. For simplicity we assumed a mean value of $\alpha$ for all radio sources in the catalogue.

The number of observed radio sources per steradian depends on the lower flux limit and can be approximated by a power law

$\frac{\mathrm{d} N}{\mathrm{~d} \Omega}(>S) \propto S^{-x}$

The value of $x$ can be different for each survey. Typically $x$ is assumed to be about one.

Two effects have to be taken into account. The emitted radio frequency $f_{\text {rest }}$ is observed at the Doppler shifted frequency $f_{\text {obs }}$.
The magnitude of this change depends on the angle $\theta$ between the direction to the source and the direction of our motion, with velocity $v$. Observed and rest frame frequencies are related by

$f_{\text {obs }}=f_{\text {rest }} \delta(v, \theta)$,

where $\delta$ is given by

$\delta(v, \theta)=\frac{1+\frac{v}{c} \cos (\theta)}{\sqrt{1-\left(\frac{v}{c}\right)^{2}}}$.

Thus the observed flux changes due to our motion, since it depends on the frequency

$S_{\text {obs }}\left(f_{\text {obs }}\right) \propto \delta f_{\text {rest }}^{-\alpha} \propto \delta^{1+\alpha} f_{\text {obs }}^{-\alpha} \propto S_{\text {rest }}\left(f_{\text {obs }}\right) \delta^{1+\alpha}$.

The first factor of $\delta$ is due to the fact that the energy of an observed photon is enhanced due to the Doppler effect.

Thus, the Doppler effect will change the number of observed sources above a given flux limit like

$\left(\frac{\mathrm{d} N}{\mathrm{~d} \Omega}\right)_{\mathrm{obs}}=\left(\frac{\mathrm{d} N}{\mathrm{~d} \Omega}\right)_{\text {rest }} \delta^{x(1+\alpha)}$.

Since the velocity of light is finite, aberration will also modify the number counts. The position of each source is changed towards the direction of motion. The new angle $\theta^{\prime}$ (observed from Earth) between the position of the source and the direction of motion is given by

$\tan \theta^{\prime}=\frac{\sin \theta \sqrt{1-\frac{v^{2}}{c^{2}}}}{\frac{v}{c}+\cos \theta}$.

Therefore, at first order in $v / c, d \Omega$ transforms like

$\mathrm{d} \Omega^{\prime}=\mathrm{d} \Omega\left(1-2 \frac{v}{c} \cos \theta\right)+O\left(\left(\frac{v}{c}\right)^{2}\right)$.

This can be combined with the Doppler effect to give the observed number density. After approximating $\delta(v, \theta)$ to first order in $\frac{v}{c}$, the result becomes

$\frac{\mathrm{d} N}{\mathrm{~d} \Omega}{ }_{\text {obs }}=\left(\frac{\mathrm{d} N}{\mathrm{~d} \Omega}\right)_{\text {rest }}\left[1+[2+x(1+\alpha)]\left(\frac{v}{c}\right) \cos (\theta)\right]$.

The amplitude of the kinetic radio dipole is then given by

$d=[2+x(1+\alpha)]\left(\frac{v}{c}\right)$.

The kinetic radio dipole points towards the direction of our peculiar motion, which in an isotropic and homogeneous Universe must also agree with the direction defined by the CMB dipole.

\subsection{Expected kinetic radio dipole}

The measured CMB dipole is $\Delta T=3.355 \pm 0.008 \mathrm{mK}$ in the direction $(l, b)=\left(263.99^{\circ} \pm 0.14^{\circ}, 48.26^{\circ} \pm 0.03^{\circ}\right)$ (Hinshaw et al. 2009). In equatorial coordinates (epoch J2000) its direction reads $(\mathrm{RA}, \mathrm{Dec})=\left(168^{\circ},-7^{\circ}\right)$. Compared to the CMB temperature of $T_{0}=2.725 \pm 0.001 \mathrm{~K}$ (Fixsen \& Mather 2002). this corresponds to a relative fluctuation of $\Delta T / T=(1.231 \pm 0.003) \times 10^{-3}$ and thus the velocity of the Solar system has been inferred from the CMB dipole to be $v=369.0 \pm 0.9 \mathrm{~km} \mathrm{~s}^{-1}$ (Hinshaw et al. 2009). 


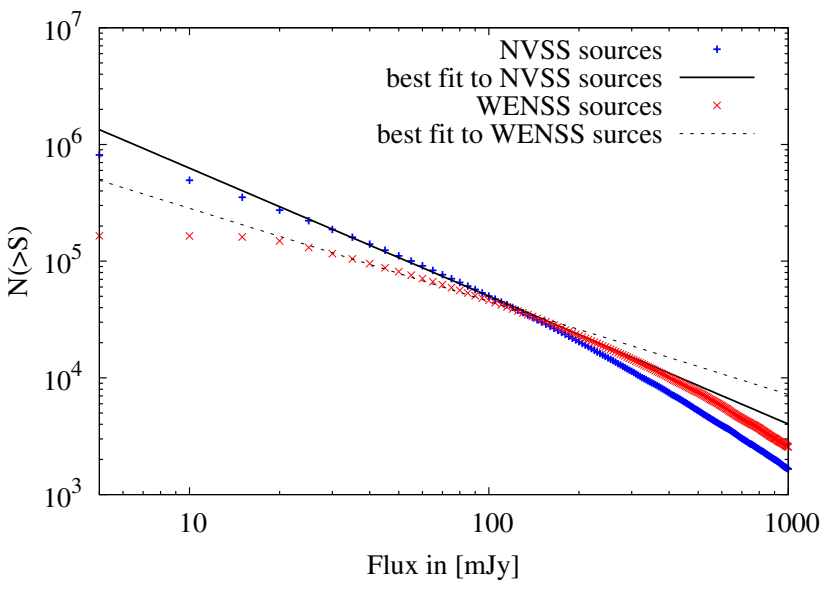

Fig. 1. Number counts of the NVSS and WENSS surveys. A function $f(S) \propto S^{-x}$ is fitted to both data sets in the range of $25 \mathrm{mJy}<S<$ $200 \mathrm{mJy}$. Resulting values of $x$ are $1.10 \pm 0.02$ for the NVSS survey and $0.80 \pm 0.02$ for the WENSS survey.

To find the expected amplitude of the kinetic radio dipole, we also need estimates for $x$ and $\alpha$. The typically assumed values are $x=1$ and $\alpha=0.75$, which gives together with $v=$ $370 \mathrm{~km} \mathrm{~s}^{-1}$ a radio dipole amplitude of $d=0.46 \times 10^{-2}$. However, we can improve on that as $x$ can be measured with help of the radio survey. Therefore we need to plot $N(>S)$ against $S$ like in Fig. 1.

For the purpose of this work we find $x_{\mathrm{NVSS}}=1.10 \pm 0.02$ and $x_{\text {WENSS }}=0.80 \pm 0.02$. The mean spectral index cannot be inferred from the catalogues, as they provide data at a single frequency band only. We thus stick to $\alpha=0.75$, but include in the dipole error an uncertainty of $\Delta \alpha=0.25$ (Garn et al. 2008). This results in the expectations:

$d_{\mathrm{NVSS}}^{\exp }=(0.48 \pm 0.04) \times 10^{-2}$,

$d_{\text {WENSS }}^{\text {exp }}=(0.42 \pm 0.03) \times 10^{-2}$.

The error is dominated by the uncertainty in the spectral index.

\section{Previous results}

The first measurement of the radio dipole using the NVSS catalogue was performed by Blake \& Wall (2002). In order to remove corruption by local structure, all sources within $15^{\circ}$ vicinity of the Galactic disk have been removed. Additionally the clustering dipole contribution was reduced by ignoring sources within $30^{\prime \prime}$ of nearby known galaxies. The spherical harmonic coefficients $a_{l m}^{\text {obs }}$ from the remaining NVSS catalogue have been determined up to $l=3$. A model for a dipole distribution with an isotropic background has been constructed $\left(a_{00}\right.$ and $\left.a_{10}\right)$. Due to masking, this dipole distribution also influences higher multipoles. After applying the same mask as for the NVSS catalogue, one finds $a_{l m}^{\text {model }}$ up to $l=3$. A quadratic estimator (chi square) was used to compare the model with the observed coefficients.

The resulting best-fit dipoles can be seen in Table 1 . The results of Blake \& Wall (2002) indicate a higher radio dipole than expected, however without statistical significance.

Singal (2011) used a linear estimator, originally proposed by Crawford (2009),

$\boldsymbol{R}_{3 \mathrm{D}}=\sum \hat{\boldsymbol{r}}_{i}$,
Table 1. Best-fit dipole parameters from Blake \& Wall (2002).

\begin{tabular}{lccccc}
\hline \hline $\begin{array}{l}\text { Flux } \\
(\mathrm{mJy})\end{array}$ & $N$ & $\begin{array}{c}\text { RA } \\
\left({ }^{\circ}\right)\end{array}$ & $\begin{array}{c}\text { Dec } \\
\left({ }^{\circ}\right)\end{array}$ & $\begin{array}{c}d \\
\left(10^{-2}\right)\end{array}$ & $\chi_{\text {red }}^{2}$ \\
\hline$>40$ & 125603 & $149 \pm 49$ & $-45 \pm 38$ & $0.7 \pm 0.5$ & 1.02 \\
$>35$ & 143524 & $161 \pm 44$ & $-27 \pm 39$ & $0.9 \pm 0.4$ & 0.74 \\
$>30$ & 166694 & $156 \pm 32$ & $2 \pm 33$ & $1.1 \pm 0.4$ & 1.01 \\
$>25$ & 197998 & $158 \pm 30$ & $-4 \pm 34$ & $1.1 \pm 0.3$ & 1.01 \\
$>20$ & 242710 & $153 \pm 27$ & $-3 \pm 29$ & $1.1 \pm 0.3$ & 1.32 \\
$>15$ & 311037 & $148 \pm 29$ & $31 \pm 31$ & $0.8 \pm 0.3$ & 1.81 \\
$>10$ & 431990 & $132 \pm 29$ & $65 \pm 19$ & $0.5 \pm 0.2$ & 4.96 \\
\hline
\end{tabular}

Notes. Coordinate system and amplitude definition are adjusted for comparison with Singal's results (see Tables 2 and 3). $N$ denotes the number of point sources with flux above the indicated limit.

Table 2. Dipole direction and amplitude from the number count estimator (15) from Singal (2011).

\begin{tabular}{lcccc}
\hline \hline $\begin{array}{l}\text { Flux } \\
(\mathrm{mJy})\end{array}$ & $N$ & $\begin{array}{c}\text { RA } \\
\left({ }^{\circ}\right)\end{array}$ & $\begin{array}{c}\text { Dec } \\
\left({ }^{\circ}\right)\end{array}$ & $\begin{array}{c}d \\
\left(10^{-2}\right)\end{array}$ \\
\hline$\geq 50$ & 91597 & $171 \pm 14$ & $-18 \pm 14$ & $2.1 \pm 0.5$ \\
$\geq 40$ & 115837 & $158 \pm 12$ & $-19 \pm 12$ & $1.8 \pm 0.4$ \\
$\geq 35$ & 132930 & $157 \pm 11$ & $-12 \pm 11$ & $1.9 \pm 0.4$ \\
$\geq 30$ & 154996 & $156 \pm 11$ & $-02 \pm 10$ & $2.0 \pm 0.4$ \\
$\geq 25$ & 185474 & $158 \pm 10$ & $-02 \pm 10$ & $1.8 \pm 0.4$ \\
$\geq 20$ & 229365 & $153 \pm 10$ & $+02 \pm 10$ & $1.8 \pm 0.3$ \\
$\geq 15$ & 298048 & $149 \pm 09$ & $+15 \pm 09$ & $1.6 \pm 0.3$ \\
\hline
\end{tabular}

and a variation of it, which we discuss below. For a large number of sources the isotropic background will clear away. The remaining vector $\boldsymbol{R}_{3 \mathrm{D}}$ will point towards the main anisotropy in the distribution of number density over the sky. To get the correct dipole amplitude $d$ one has to normalize this estimator depending on the number of sources. In Singal's analysis sources within $10^{\circ}$ of the Galactic plane have been removed. In order to avoid directional bias (see the more detailed discussion below), he reestablished a north-south symmetry of the NVSS by cutting all sources with dec $>40^{\circ}$. The results of Singal (2011) are shown in Table 2. The errors of the directional measurements are quite small here. This is an effect of an unexpectedly large amplitude, which simplifies the measurement. While the direction agrees with the one found by Blake \& Wall (2002), the dipole amplitude seems to be a factor of about four higher than expected from the CMB dipole and twice as big as found by Blake \& Wall (2002).

Masking the supergalactic plane in order to reduce the contribution of local structure did not resolve the discrepancy. Since unknown clustering further away from the super Galactic plane could also have contributed to the measurement, a second test was performed. A clustering contribution to the dipole would not give a signal proportional to $\cos \theta$. On the other hand, the difference in number counts of areas that are opposite to each other should decrease with $\cos \theta$ (where $\theta$ is the angle between an area and the measured dipole direction), if the measured dipole is due to our velocity. Singal was able to fit such a behaviour to the data. Therefore he concludes that the radio dipole amplitude is not due to local clustering.

Singal (2011) also used a linear estimator for the distribution of flux over the sky. This estimator is similar to the number density estimator (15), but weights each radio source by its flux $S_{i}$,

$\boldsymbol{R}_{\text {flux }}=\sum S_{i} \hat{\boldsymbol{r}}_{i}$ 
Table 3. Dipole direction and amplitude from the flux weighted number count estimator (16) from Singal (2011).

\begin{tabular}{lcccc}
\hline \hline $\begin{array}{l}\text { Flux } \\
(\mathrm{mJy})\end{array}$ & $N$ & $\begin{array}{c}\text { RA } \\
\left({ }^{\circ}\right.\end{array}$ & $\begin{array}{c}\text { Dec } \\
\left({ }^{\circ}\right)\end{array}$ & $\begin{array}{c}d \\
\left(10^{-2}\right)\end{array}$ \\
\hline $1000>S \geq 50$ & 90360 & $163 \pm 12$ & $-11 \pm 11$ & $2.3 \pm 0.7$ \\
$1000>S \geq 40$ & 114600 & $159 \pm 12$ & $-11 \pm 11$ & $2.2 \pm 0.6$ \\
$1000>S \geq 35$ & 131691 & $159 \pm 11$ & $-10 \pm 10$ & $2.2 \pm 0.6$ \\
$1000>S \geq 30$ & 153759 & $159 \pm 11$ & $-07 \pm 10$ & $2.2 \pm 0.6$ \\
$1000>S \geq 25$ & 184237 & $159 \pm 10$ & $-07 \pm 09$ & $2.2 \pm 0.6$ \\
$1000>S \geq 20$ & 228128 & $158 \pm 10$ & $-06 \pm 09$ & $2.1 \pm 0.5$ \\
$1000>S \geq 15$ & 296811 & $157 \pm 09$ & $-03 \pm 08$ & $2.0 \pm 0.5$ \\
\hline
\end{tabular}

Like $\boldsymbol{R}_{3 \mathrm{D}}$, this estimator finds the main anisotropy and the amplitude needs to be normalized. The brightest sources $(S>$ $1000 \mathrm{mJy}$ ) are removed, because they would dominate $\boldsymbol{R}_{\text {flux }}$ otherwise. Results of this estimator are shown in Table 3. The estimated directions are in agreement with the results of Blake \& Wall (2002) and the number count estimator results of Singal (2011). However, the normalized dipole amplitudes $d$ are even higher than those of the number count estimator $\boldsymbol{R}_{3 \mathrm{D}}$. In Sect. 6 we resolve this conflict.

Most recently, Gibelyou \& Huterer (2012) measured a dipole amplitude $(d=2.7 \pm 0.5) \times 10^{-2}$ towards $(\mathrm{RA}, \mathrm{Dec})=(117 \pm$ $\left.20^{\circ}, 6 \pm 14^{\circ}\right)$ from the NVSS. This direction is inconsistent with the studies mentioned above and the dipole amplitude is a factor of five larger than expected. The authors used separate estimators for the direction and the amplitude. Their direction estimate is based on a linear estimator, originally proposed by Hirata (2009),

$\boldsymbol{R}_{3 \mathrm{DM}}=\sum_{i}^{N_{\mathrm{D}}} \hat{r}_{i}-\frac{N_{\mathrm{D}}}{N_{\mathrm{R}}} \sum_{j}^{N_{\mathrm{R}}} \hat{r}_{j}$.

This three-dimensional estimator (3DM) is intended to be unbiased for arbitrary survey geometries and arbitrary masking. The idea is to achieve that with help of the second sum, which goes over $N_{\mathrm{R}}$ randomly distributed points, subject to the same masking. Therefore, the authors include all sources of the NVSS survey, except for those within $10^{\circ}$ of the Galactic plane. Below we show that this estimator has a direction bias, which depends on the real dipole anisotropy.

We summarize, there is no agreement on the amplitude and direction of the cosmic radio dipole so far.

\section{Linear estimators for a full sky}

Let us first show that the estimator (15) provides an unbiased estimate of the dipole direction.

Starting from the distribution of the number of radio sources per solid angle (11), as seen by a moving observer in an otherwise isotropic Universe, the probability to find a given radio source within a solid angle $d \Omega$ of position $\hat{\boldsymbol{r}}$ is given by

$p(\hat{\boldsymbol{r}}) \mathrm{d} \Omega=\frac{1}{4 \pi}(1+\hat{\boldsymbol{r}} \cdot \boldsymbol{d}) \mathrm{d} \Omega$,

where $\boldsymbol{d}$ denotes the dipole vector.

To study the bias of an estimator, we calculate its expectation value with respect to an ensemble average. We do so below by means of Monte Carlo studies. For analytic considerations, for large $N$ we replace the ensemble average by a spatial average, i.e.

$\langle 1\rangle=\int \prod_{i=1}^{N} \mathrm{~d} \Omega_{i} p\left(\hat{\boldsymbol{r}}_{i}\right) 1=1$,

thus we assume ergodicity. Note that the average is a linear operator.

Now the expectation value of Crawford's estimator can be evaluated for large $N$,

$\left\langle\boldsymbol{R}_{3 \mathrm{D}}\right\rangle=\left\langle\sum_{i=1}^{N} \hat{r}_{i}\right\rangle=\sum_{i=1}^{N}\left\langle\hat{r}_{i}\right\rangle=\frac{N}{4 \pi} \int \mathrm{d} \Omega(1+\hat{r} \cdot \boldsymbol{d}) \hat{r}$.

This calculation holds for independent, identically distributed positions $\hat{r}_{i}$, thus without clustering effects. Only the second term survives the integration and thus the expected dipole estimator is

$\left\langle\boldsymbol{R}_{3 \mathrm{D}}\right\rangle=\frac{1}{3} N \boldsymbol{d}$.

Naively, one could now estimate the dipole signal by $\boldsymbol{d}_{3 \mathrm{D}} \equiv$ $\frac{3}{N} \boldsymbol{R}_{3 \mathrm{D}}$.

We conclude that $\boldsymbol{d}_{3 \mathrm{D}}$ provides us with an unbiased estimate of the dipole direction $\hat{\boldsymbol{d}}$ for a full sky sample. However the estimated dipole amplitude $\left|\boldsymbol{d}_{3 \mathrm{D}}\right|$ is biased.

To understand the origin of this bias let us first consider

$\left\langle\boldsymbol{d}_{3 \mathrm{D}}^{2}\right\rangle=\left(1-\frac{1}{N}\right) d^{2}+\frac{9}{N}>d^{2}$.

The inequality holds for large $N$ and $d<3$ (in case of large dipole amplitudes $[d=O(1)]$ our ansatz (19) should also take many-point correlations into account). Thus $\boldsymbol{d}_{3 \mathrm{D}}^{2}$ is definitely biased towards higher amplitudes. However, to prove that $\left|\boldsymbol{d}_{3 \mathrm{D}}\right|$ is biased, we would need to calculate $\left\langle\left|\boldsymbol{d}_{3 \mathrm{D}}\right|\right\rangle$. We do this by means of the random walk/flight method.

\subsection{Random flight}

Adding up vectors for each point of a survey corresponds to a random walk with unit step size. To be more precise this is a random flight, since the problem is three dimensional. Even for a vanishing dipole, such a random flight is unlikely to return to the origin after $N$ steps. This describes the noise of any realisation of an isotropic distribution of $N$ sources.

Following Crawford (2009), we determine the distance $r$ from the origin after $N$ steps from the probability density of a random flight process

$\check{P}_{N}(r) \mathrm{d} r=\left[\frac{54}{\pi N^{3}}\right]^{1 / 2} r^{2} \exp \left(-\frac{3 r^{2}}{2 N}\right) \mathrm{d} r$.

The probability of measuring a dipole signal of an amplitude bigger than $R$ in a random flight is

$P_{N}\left(R>R_{p C L}\right)=\int_{R_{p C L}}^{\infty} \mathrm{d} r \check{P}_{N}(r)=1-p C L$.

A confidence level $p C L$ can be choosen, leading to errorbars for a measured dipole vector $R_{3 \mathrm{D}} \pm R_{p C L}$. To estimate the directional uncertainties of this method, Crawford (2009) made the following argument: at a given confidence level the random flight corresponds to a step of length up to $R_{p C L}$. Adding $R_{p C L}$ 


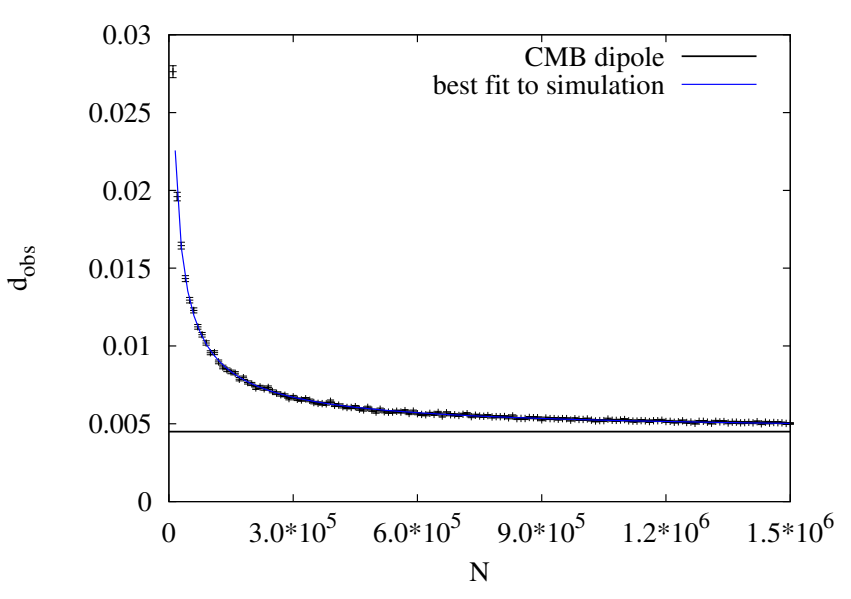

Fig. 2. Ampliude bias of the full sky estimator $d_{3 \mathrm{D}}$. Data represent mean and empirical variance of 1000 simulations for each $N$. A function $d_{\text {obs }}(N)=\sqrt{D^{2}+9 A^{2} / N}$ is fitted to the simulated data, with best-fit values $A=0.908 \pm 0.002, D=(0.451 \pm 0.001) \times 10^{-2}$. The expected dipole amplitude $(d=0.0046)$ is indicated by the horizontal line.

perpendicular to the measured dipole $\boldsymbol{R}_{3 \mathrm{D}}$ allows us to estimate the maximal offset in direction. Using trigonometry, one can relate $R_{p C L}$ to the directional uncertainties expressed as the angle

$\delta \theta_{p C L}=\arcsin \frac{R_{p C L}}{R_{3 \mathrm{D}}}$.

The expected magnitude of the random dipole contribution is estimated from (23) as

$\left\langle R_{3 \mathrm{D}}^{\text {random }}\right\rangle=\int_{0}^{\infty} r\left[\frac{54}{\pi N^{3}}\right]^{1 / 2} r^{2} \exp \left(-\frac{3 r^{2}}{2 N}\right) \mathrm{d} r \approx 0.92 \sqrt{N}$.

Since the random dipole has no distinguished direction, there is no direction bias of the linear estimator for a full sky map.

Even for vanishing $d$, this gives rise to a non-vanishing estimate of the dipole amplitude $d_{3 \mathrm{D}}^{\text {random }}=2.8 N^{-1 / 2}$ and is thus the origin of the amplitude bias.

Motivated by (22) we make the following ansatz for the dipole amplitude and its bias:

$\left\langle d_{3 \mathrm{D}}\right\rangle=\sqrt{d^{2}+\frac{9}{N^{2}}\left\langle R_{3 \mathrm{D}}^{\text {random }}\right\rangle^{2}}$.

To verify this analytic estimate of the biased amplitude, we simulated full sky maps including a velocity dipole (with $v=$ $370 \mathrm{~km} \mathrm{~s}^{-1}$ ). From these simulated catalogues we extracted the observed amplitude $d_{\text {obs. }}$. Figure 2 shows the simulated data, the true value of the dipole amplitude and a fit of the form $f(N)=$ $\sqrt{D^{2}+9 A^{2} / N}$.

The best-fit values are $A=0.908 \pm 0.002$ and $D=(0.451 \pm$ $0.001) \times 10^{-2}$ (statistical errors only). These numbers should be compared to the factor 0.92 from (26) and the simulation input of $d=0.46 \times 10^{-2}$. The reduced chi- square of the fit is $7 \times 10^{-5}$. Thus the Monte Carlo simulations agree with the theoretically motivated ansatz (27) for the expected dipole amplitude of the estimator $\boldsymbol{d}_{3 \mathrm{D}}$.

We conclude that even for a perfect full sky catalogue (no masking, complete in flux, perfect flux and position measurements), the amplitude of the linear estimator is biased towards higher values. Increasing the number of radio sources will reduce the bias. The estimator $d_{3 \mathrm{D}}$ is asymptotically unbiased, but this is of limited practical use for the analysis of NVSS and WENSS data. A similar bias of the dipole amplitude is also found for the other linear estimators introduced above. This finding is in agreement with Gibelyou \& Huterer (2012), who use a linear estimator to find the direction of the dipole.

\section{Linear estimators for an incomplete sky}

So far we assumed full coverage of the radio sky. More realistic catalogues cover just a fraction of the sky, as all earth based telescopes are limited to observe at certain declination ranges. Additionally, the Milky Way is masking parts of the sky. Here we discuss some of the effects caused by incomplete sky coverage.

The upcoming Low Frequency Array (LOFAR) Tier-1 survey will cover about half of the sky $(2 \pi)$, thus we first focus on this situation. As a second step we generalize this to an arbitrary axisymmetric survey geometry and include the effects of masking.

\subsection{Random walk}

Let us assume a survey geometry that covers all of the Northern hemisphere and ask how the estimator of the radio dipole (15) has to be modified. For the first two Cartesian components of $\boldsymbol{R}_{3 \mathrm{D}}$ there should be no systematic problem, but the third component will definitely be biased. It is necessary to remove the effect of the incomplete sky from this $z$ component. Consider the expectation value of $\boldsymbol{R}_{3 \mathrm{D}}$ for the Northern hemisphere

$\left\langle\boldsymbol{R}_{3 \mathrm{D}}\right\rangle_{\text {hemisphere }}=\left\langle\sum_{i=1}^{N} \hat{r}_{i}\right\rangle=\frac{N p_{\mathrm{d}}}{2 \pi} \int_{\delta>0} \mathrm{~d} \Omega(1+\hat{r} \cdot \boldsymbol{d}) \hat{r}$,

where $\left.p_{\mathrm{d}} \equiv 1 /\left[1+(d / 2) \cos \vartheta_{\mathrm{d}}\right)\right]$ accounts for the proper normalisation of the probability distribution on the hemisphere in presence of a dipole. $4 \pi$ in (18) becomes $2 \pi$ for obvious reasons. The integral can be evaluated by hand. One finds

$\left\langle\boldsymbol{R}_{3 \mathrm{D}}\right\rangle_{\text {hemisphere }}=N p_{\mathrm{d}}\left(\begin{array}{c}\frac{1}{3} d \cos \varphi_{\mathrm{d}} \sin \vartheta_{\mathrm{d}} \\ \frac{1}{3} d \sin \varphi_{\mathrm{d}} \sin \vartheta_{\mathrm{d}} \\ \frac{1}{2}+\frac{1}{3} d \cos \vartheta_{\mathrm{d}}\end{array}\right)$,

where $\vartheta_{\mathrm{d}}$ and $\varphi_{\mathrm{d}}$ denote the dipole position in spherical coordinates. The $z$ direction is strongly influenced by the incomplete sky coverage. The total number of observed sources is not independent from the amplitude and orientation of the dipole itself.

Nevertheless there is no problem in the evaluation of $\varphi_{\mathrm{d}}$ because one can calculate

$\varphi_{\mathrm{d}}=\arctan \frac{R_{y}}{R_{x}}$.

Here $N$ as well as $\sin \vartheta_{\mathrm{d}}$ cancel out. So the 2D estimator is unbiased with respect to $\varphi_{\mathrm{d}}$. Therefore we propose a pure two dimensional estimator:

$\boldsymbol{R}_{2 \mathrm{D}}=\sum_{i}^{N}\left(\begin{array}{c}\cos \varphi_{i} \sin \vartheta_{i} \\ \sin \varphi_{i} \sin \vartheta_{i} \\ 0\end{array}\right)$

From this one can still use $\left(R_{x}^{2}+R_{y}^{2}\right)^{-1 / 2}$ for evaluating $d \sin \vartheta_{\mathrm{d}} N p_{\mathrm{d}} / 3$ and $\varphi_{\mathrm{d}}$. Let us take a look at the factor $\sin \vartheta_{\mathrm{d}}$. Sources near the pole will make a smaller contribution than those further away. If a source near the pole is shifted by a small distance, the value $\varphi_{i}$ of this source could change dramatically. 
So the weighting terms compensate for this artificially big errors, which are just a relic of the coordinate system.

Let us now estimate the uncertainties of the estimator $\boldsymbol{R}_{2 \mathrm{D}}$. The problem corresponds to an isotropic random walk process with variable step size. The probability density for a displacement of $r$ for such a random walk is

$\check{P}_{N}(r)_{2 \mathrm{D}} \mathrm{d} r=\frac{3}{N} r \exp \left(-\frac{3 r^{2}}{2 N}\right) \mathrm{d} r$.

Similar to the random flight we determine $R_{p C L}$ defined by

$P_{N}\left(R_{2 \mathrm{D}}>R_{p C L}\right)=\int_{R_{p C L}}^{\infty} \mathrm{d} r \check{P}_{N}(r)_{2 \mathrm{D}}=1-p C L$.

Here again $p C L$ is the confidence level. It is possible to solve the above integral analytically

$\int_{R_{p C L}}^{\infty} \mathrm{d} r \frac{3}{N} r \exp \left(-\frac{3 r^{2}}{2 N}\right)=\exp \left(-\frac{3 R_{p C L}^{2}}{2 N}\right)$.

So $R_{p C L}$ is given by

$R_{p C L}=\sqrt{\frac{2 N}{3} \ln \left(\frac{1}{1-p C L}\right)}$

Now one can use the same argument as for the random flight to evaluate the uncertainty of the $\varphi_{\mathrm{d}}$ estimation

$\delta \varphi_{p C L}=\arcsin \frac{R_{p C L}}{R_{2 \mathrm{D}}}$.

In this way one can directly determine error bars for measured results of $\varphi_{\mathrm{d}}$ calculated via (30). Using this two dimensional estimator one cannot measure the dipole amplitude $d$ itself but only the combination $d \sin \vartheta_{\mathrm{d}} N p_{\mathrm{d}} / 3$. Therefore it can only give an lower limit for $d$.

Like in the case of the full three dimensional estimator, this version is also biased in the measurement of the amplitude. The expectation of the random contribution can be calculated via

$\left\langle R_{2 \mathrm{D}}^{\text {random }}\right\rangle=\int_{0}^{\infty} r \frac{3}{N} r \exp \left(-\frac{3 r^{2}}{2 N}\right) \mathrm{d} r \approx 0.72 \sqrt{N}$.

So we expect our estimator to measure a combination of this random contribution and the true velocity dipole and make the ansatz

$\left\langle d_{2 \mathrm{D}}\right\rangle=\sqrt{d^{2} \sin ^{2} \vartheta_{\mathrm{d}}+\frac{9}{N^{2}}\left\langle R_{2 \mathrm{D}}^{\text {random }}\right\rangle^{2}}$,

where we used $p_{\mathrm{d}} \approx 1$. Like above, we verify this via Monte Carlo simulations, shown in Fig. 3.

\subsection{Direction bias}

For any masked or incomplete map of the sky, we cannot measure the mean source density $\bar{N} /(4 \pi)$, i.e. the monopole. Therefore we always have to keep in mind that the observed mean density is just an approximation. Gibelyou \& Huterer (2012) have used an estimator proposed by Hirata (2009), which implicitly assumes, that the monopole is known. Based on the knowledge of the monopole, this estimator would compensate for masking effects and incomplete sky coverage by substracting a pure random isotropic map from the observed dipole term via (17). However, we cannot know $\bar{N}$.

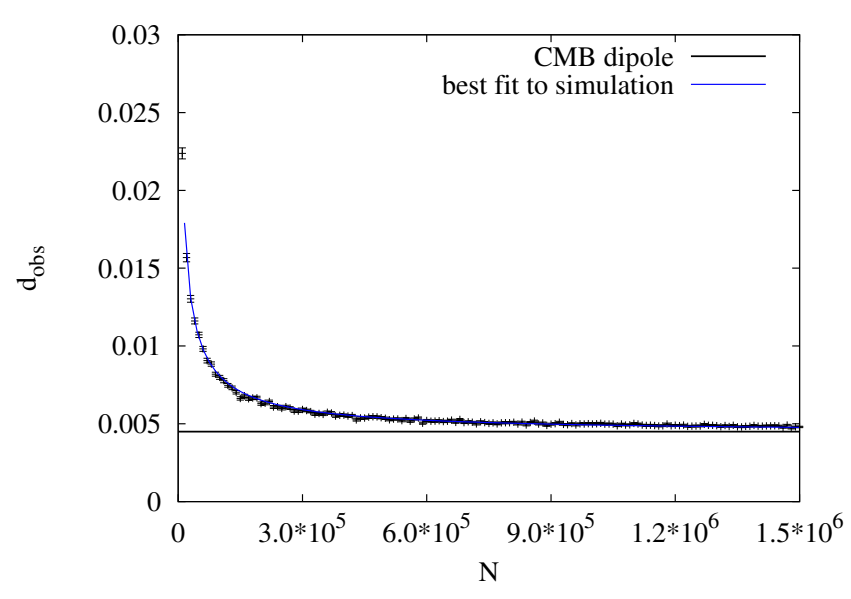

Fig. 3. Ampliude bias for the estimator $d_{2 \mathrm{D}}$ on a hemisphere. Data represent mean and empirical variance of 1000 simulations for each N. A function $d_{\mathrm{obs}}(N)=\sqrt{D^{2}+9 A^{2} / N}$ is fitted to the simulated data, with best fit values $A=0.712 \pm 0.003$ and $D=(0.444 \pm 0.002) \times 10^{-2}$. The dipole amplitude $(0.0046)$ is indicated by the horizontal line, the dipole vector is assumed to lie in the equatorial plane $\left(\sin \vartheta_{\mathrm{d}}=1\right)$.

For the previous example of a hemisphere, the monopole density is $\bar{N} /(4 \pi)=N p_{\mathrm{d}} /(2 \pi)$, where $N=N_{\mathrm{D}}$ in (17). The random map can be made up of an arbitrary number of sources $N_{\mathrm{R}}$, but is reweighted by the observed number of sources $N_{\mathrm{D}} / N_{\mathrm{R}}$, instead of $\bar{N} / N_{\mathrm{R}}$. This introduces a directional bias, in addition to the previously discussed biased amplitude. One can see this explicitly by applying (17) on our model of a dipole modified isotropic hemisphere. We find

$\left\langle\boldsymbol{R}_{3 \mathrm{DM}}\right\rangle_{\text {hemisphere }}=\frac{N p_{\mathrm{d}}}{3} d\left(\begin{array}{c}\cos \varphi_{\mathrm{d}} \sin \vartheta_{\mathrm{d}} \\ \sin \varphi_{\mathrm{d}} \sin \vartheta_{\mathrm{d}} \\ \cos \vartheta_{\mathrm{d}}\end{array}\right)+\frac{1}{2} N\left(\begin{array}{c}0 \\ 0 \\ p_{\mathrm{d}}-1\end{array}\right)$,

which clearly is not parallel to $\boldsymbol{d}$. For small values of $d$ we can expand $p_{\mathrm{d}}$ and obtain

$\left\langle\boldsymbol{R}_{3 \mathrm{DM}}\right\rangle_{\text {hemisphere }}=\frac{N}{3} d\left(\begin{array}{c}\cos \varphi_{\mathrm{d}} \sin \vartheta_{\mathrm{d}} \\ \sin \varphi_{\mathrm{d}} \sin \vartheta_{\mathrm{d}} \\ \frac{1}{4} \cos \vartheta_{\mathrm{d}}\end{array}\right)+O\left(d^{2}\right)$.

The $z$ component of the dipole is underestimated by a factor of 4 for the geometry of a hemisphere. Despite cancelation of the leading term of the bias of the $z$ direction, the dipole direction remains biased. Less symmetric survey geometries lead to a bias of all dipole components for this estimator.

The best strategy to avoid any directional bias for a linear estimator is to make the survey geometry point symmetric around the observer for three dimensional estimators like $\boldsymbol{R}_{3 \mathrm{D}}$ or point symmetric around the zenith in case of two dimensional estimators $\boldsymbol{R}_{2 \mathrm{D}}$. This implies for the NVSS that one has to cut symmetric in declination, such that both polar caps are missing, a strategy that was used by Singal (2011).

\subsection{Masking}

The use of a masked sky additionally affects the dipole measurement. In general, the estimated dipole direction and amplitude depend on the position of the true dipole relative to the mask. Cutting areas with large dipole contribution will reduce the amplitude and vice versa. 
In the following we consider masks that are point symmetric with respect to the observer for all considered 3-dimensional estimators, respectively point symmetric with respect to the zenith for the 2-dimensional estimators. Constructed in such a way, masking does not introduce any directional bias. Nevertheless, we have to consider the effects of masking on the estimated dipole amplitude.

A simple method to correct for this effect was put forward by Singal (2011), who introduced a masking factor $k$,

$\boldsymbol{R}_{3 \mathrm{D}}^{\mathrm{mask}}=\frac{1}{k_{3 \mathrm{D}}} \sum \boldsymbol{r}_{i}$

Such a factor is expected to be a function of shape and position of the mask as well as the dipole position. In most cases an analytic calculation of this factor is impossible. For simple mask geometries some analytic results can be found in Rubart (2012). An alternative approach is to simulate the effects by means of Monte Carlos and to compare simulations with and without masking. The ratio of both results after a large number of simulations provides the masking factor $k_{3 \mathrm{D}}$.

Doing so, we found some interesting effects. The masking factors depend on the number of objects in the simulated maps as well as on the true dipole amplitude. This can be explained as follows. The masking will mainly affect the kinetic dipole contribution, while the random dipole will increase due to the decrease of the number of objects in the masked catalogue. Therefore we expect the amplitude to be

$\left\langle d_{3 \mathrm{D}}\right\rangle_{\mathrm{mask}}=\sqrt{k_{3 \mathrm{D}}^{2} d^{2}+\frac{9}{N^{2}}\left\langle R_{3 \mathrm{D}}^{\text {random }}\right\rangle^{2}}$.

$k_{3 \mathrm{D}}$ should only depend on the properties of the mask and not on the amplitude of the dipole. To test this, we created simulated maps for two different dipole amplitudes, with values motivated by the CMB measurement $d_{\mathrm{CMB}}=0.46 \times 10^{-2}$ and by the measurement of Blake \& Wall (2002) at $25 \mathrm{mJy} d_{\mathrm{BW}}=1.1 \times 10^{-2}$. We used the direction RA $=158^{\circ}, \mathrm{Dec}=-4^{\circ}$ in both cases. The mask agrees with the one used by Singal (2011), i.e. we removed all sources with $|\delta|>40^{\circ}$ and $|b|<10^{\circ}$. Resulting amplitudes for different numbers of sources are shown in Fig. 4.

First of all we can conclude that (42) is a good fit for the behaviour of the simulated maps in both cases. The measured amplitudes are larger than those estimated from full sky maps. We can now calculate $k_{3 \mathrm{D}}$. In both cases it turns out to be 1.4, which could be used to correct the amplitude estimate.

A similar argument holds for the two dimensional estimator. Here we expect a behaviour of the form:

$\left\langle d_{2 \mathrm{D}}\right\rangle_{\text {mask }}=\sqrt{k_{2 \mathrm{D}}^{2} d^{2} \sin ^{2} \vartheta_{\mathrm{d}}+\frac{9}{N^{2}}\left\langle R_{2 \mathrm{D}}^{\text {random }}\right\rangle^{2}}$.

Using the same assumptions about the dipole term and the same mask as before, we obtain Fig. 5 .

This time, we find $k_{2 \mathrm{D}}=1.3$ for both assumed velocities. The simulations support our assumption that the masking factor does not depend on the dipole magnitude $d$.

\subsubsection{Masking correction}

Although the masking factor $k$ does not depend on the amplitude $d$, it may depend on the dipole direction $\hat{\boldsymbol{d}}$. Therefore it would be necessary to repeat the analysis of the previous section for each dipole direction found. To reduce the simulation effort, we rely on the following method instead.

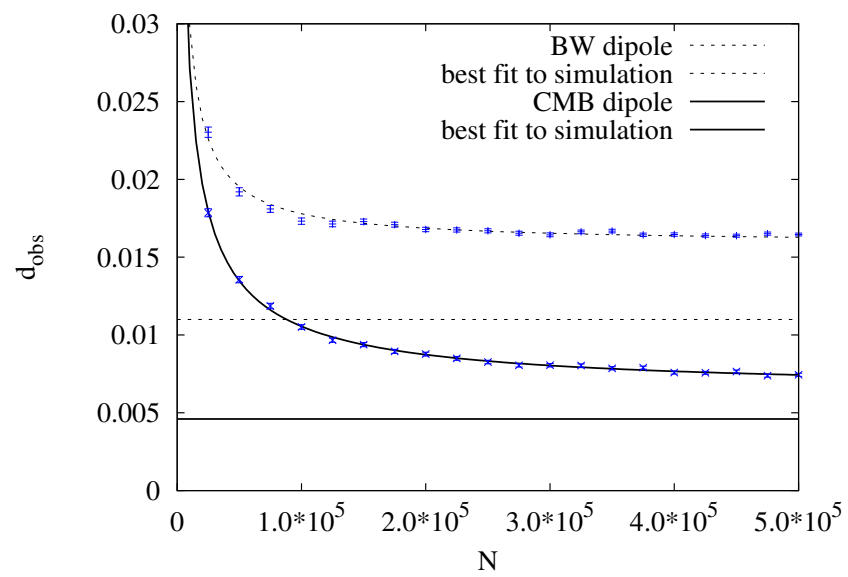

Fig. 4. Amplitude bias of the 3-dimensional estimator for the masked NVSS geometry of Singal (2011). Data represent mean and empirical variance of 1000 simulations for each $N$. A function $d_{\mathrm{obs}}(N)=$ $\sqrt{(K D)^{2}+9 A^{2} / N}$ is fitted to the simulated data, with best-fit values $A=$ $0.883 \pm 0.006, K D=(0.642 \pm 0.005) \times 10^{-2}$ and $A=0.847 \pm 0.016$, $K D=(1.59 \pm 0.008) \times 10^{-2}$ for the expected kinetic radio dipole and the radio dipole measured by Blake \& Wall (2002), respectively. The simulated dipole amplitudes, without masking, are indicated by the horizontal lines.

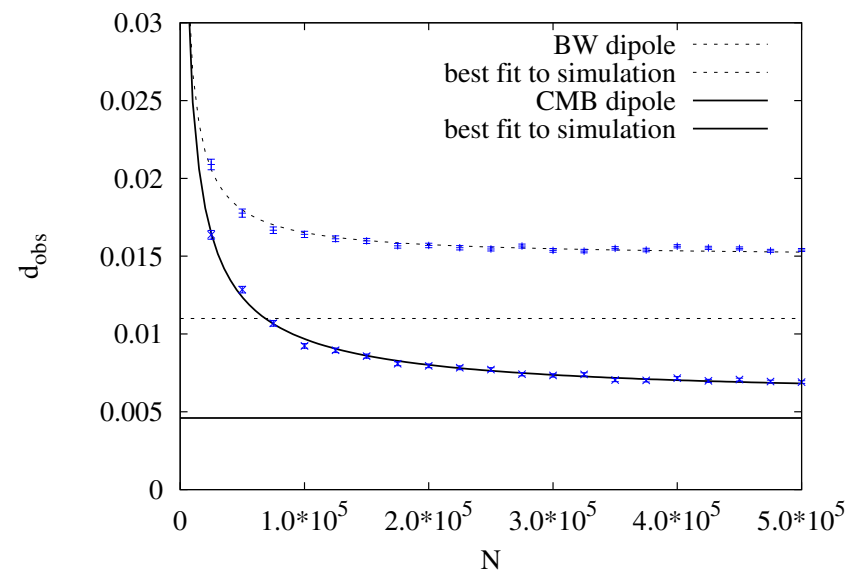

Fig. 5. Amplitude bias of the 2-dimensional estimator for the masked NVSS geometry of Singal (2011). Data represent mean and empirical variance of 1000 simulations for each $N$. A function $d_{\text {obs }}(N)=$ $\sqrt{(K D)^{2}+9 A^{2} / N}$ is fitted to the simulated data, with best-fit values $A=$ $0.810 \pm 0.009, K D=(0.589 \pm 0.008) \times 10^{-2}$ and $A=0.745 \pm 0.014$, $K D=(1.493 \pm 0.006) \times 10^{-2}$, for the expected kinetic radio dipole and the dipole measured by Blake \& Wall (2002), respectively. The simulated dipole amplitudes, without masking, are indicated by the horizontal lines.

For the full, as well as for the masked sky, surveys with $10^{6}$ sources were simulated. This choice guarantees that we investigate masking effects and not effects due to shot noise. The mean dipole amplitudes are determined for $10^{3}$ simulated full and masked sky surveys, respectively. The ratio of the masked sky mean amplitude to the full sky mean amplitude is denoted $\tilde{k}$. This ratio provides a first approximation to the masking factor.

$\tilde{k} \equiv \frac{\sqrt{k^{2} d^{2}+\frac{9}{N^{2}}\left\langle R^{\text {random }}\right\rangle^{2}}}{\sqrt{d^{2}+\frac{9}{N^{2}}\left\langle R^{\text {random }}\right\rangle^{2}}}$.

The influence of the random dipole tends to bias $\tilde{k}$ towards 1 (as can be easily seen in the limit of a small number of sources). 
This bias can be compensated by rewriting the above formula into

$k=\sqrt{\tilde{k}^{2}+\frac{9\left\langle R^{\text {random }}\right\rangle^{2}}{d^{2} N^{2}}\left(\tilde{k}^{2}-1\right)}$.

The values of $N$ and $d$ are input parameters of the simulation. From the last section we know the values of $\left\langle R^{\text {random }}\right\rangle$ for the three- as well as for the two-dimensional case. Therefore we can transform the approximated masking factor $\tilde{k}$ into the unbiased masking factor $k$.

\section{Dipole from a flux weighted estimator}

Let us now turn to a discussion of the flux weighted estimator (16), which was used by Singal (2011).

We first take a closer look at the theoretically expected value of the flux dipole $\boldsymbol{d}_{\text {flux }}$. For simplicity we assume full sky coverage. For a large number of sources,

$\hat{\boldsymbol{d}} \cdot \sum_{i=1}^{N} S_{i} \boldsymbol{r}_{i} \approx \int_{4 \pi} \mathrm{d} \Omega \int_{S_{\min }}^{S_{\max }} \mathrm{d} S \frac{\mathrm{d}^{2} N}{\mathrm{~d} \Omega \mathrm{d} S} S \cos \theta$,

where $\theta$ is the angle between a source and the dipole direction $\hat{\boldsymbol{d}}$.

We now determine the number of sources per flux and solid angle as a function of the observer velocity. At zeroth order in velocity, this density $n(S)$ is isotropic. As for the number counts, stellar aberration and the Doppler effect have to be taken into account. Stellar aberration gives rise to

$$
\frac{\mathrm{d}^{2} N}{\mathrm{~d} \Omega \mathrm{d} S} \approx n(S)\left(1+2 \frac{v}{c} \cos \theta\right) \text {. }
$$

The relativistic Doppler effect alters the observed fluxes. When we observe a source in the direction of motion, we measure a higher flux than if we were at rest with respect to the isotropic and homogeneous Universe. We relate the observed flux $S_{0}$ to the flux that is measured by an observer with vanishing peculiar motion,

$S_{\text {rest }} \approx S_{0}-S_{0}(1+\alpha) \frac{v}{c} \cos \theta$.

We assume the power law $n(S)=a S^{-\tilde{x}}$ to hold for observers at rest and Taylor expand around the observed flux

$n\left(S_{\text {rest }}\right) \approx n\left(S_{0}\right)\left[1+\tilde{x}(1+\alpha) \frac{v}{c} \cos \theta\right]$.

If the assumed power law holds for all sources of a survey, then $\tilde{x}=1+x$, with $x$ as defined above in (4). Combining the Doppler effect and the stellar aberration leads to

$$
\frac{\mathrm{d}^{2} N}{\mathrm{~d} \Omega \mathrm{d} S}=n_{0}\left(S_{0}\right)\left[1+(2+\tilde{x}(1+\alpha)) \frac{v}{c} \cos \theta\right]+O\left(\left(\frac{v}{c}\right)^{2}\right) .
$$

This result only holds under the assumption of a power law behaviour of the number counts. It is crucial to keep this in mind.

\subsection{NVSS}

Let us now see, if we are allowed to make this assumption for the analysis of NVSS data. The plot in Fig. 6 demonstrates that the power law is not valid for the flux range used in the analysis of Singal (2011), i.e. fluxes up to 1 Jy. Actually the slope steepens for the larger fluxes considered. The best fit power-law gives

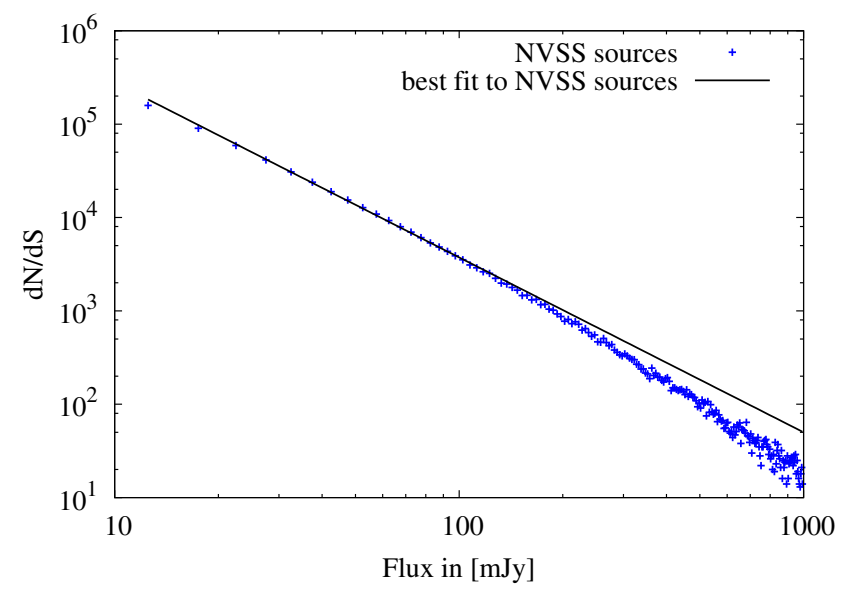

Fig. 6. Differential number counts of the NVSS catalogue, $S_{\min }=$ $10 \mathrm{mJy}$, best fit values for $f(s)=a \cdot s^{-\tilde{x}}$ with $25 \mathrm{mJy}<S<1000 \mathrm{mJy}$ are $a=2.1 \times 10^{7}$ and $\tilde{x}=1.9$.

a reduced chi-squared value of $\chi^{2}=122$. That means that the observed data cannot be fitted by a power law.

Thus Singal's assumption $\tilde{x} \approx 1$ does not hold for two reasons. Firstly, for a pure power law we would expect $\tilde{x}=1+x \approx 2$, which is close to what we find: $\tilde{x} \approx 1.9$. Secondly, the power-law assumption only applies to about one half of the data.

In conclusion, the result of unexpectedly large amplitudes in Singal (2011) could partly be explained by this two effects. With power law behaviour one should use $\tilde{x}=1.9$ or larger to also account for the steepening of the spectral index at high fluxes, which increases the expectation value by

$\frac{\left.\left\langle\boldsymbol{d}_{\mathrm{obs}}\right\rangle\right|_{\tilde{x}=1.9}}{\left.\left\langle\boldsymbol{d}_{\mathrm{obs}}\right\rangle\right|_{x=1}}>1.4$.

The results of the flux weighted estimator from Singal (2011) should be reduced by at least a factor of 1.4.

Compared to the estimator of Crawford, the estimator (16) stresses sources with high flux. To avoid the domination of a small number of sources, sources with $S>1000 \mathrm{mJy}$ are not taken into account. Nevertheless, this estimator is stressing bright sources. These sources are, on average, nearer than the rest. Hence (16) might be dominated by nearby sources and by atypically bright ones. This seems to be yet another weakness of this estimator, since the local universe is anisotropic.

\section{Dipole estimates from NVSS and WENSS}

\section{1. $3 D$ linear estimates}

We are now in a position to check the three dimensional estimations of the radio dipole in the NVSS catalogue. As we have shown above, the estimator used by Gibelyou \& Huterer (2012) (17) gives rise to a biased dipole direction and thus is not further considered in this work. The flux weighted estimator (16) is also of limited use, as the NVSS data cannot be described by a power-law over all fluxes of interest. We thus focus here on the simplest linear estimator (15).

In order to obtain an unbiased direction estimate, the cut sky geometry of Singal (2011) is adopted. The masking factor $k$ is determined for every measured dipole anisotropy direction, as described in 5.3.1. The pure estimator results $d_{3 \mathrm{D}}$ are then corrected for the masking bias and we obtain $d_{3 \mathrm{D}}^{\text {cor }}$.

All results for right ascension agree within their error bars. The same holds true for the dipole amplitudes. This 
Table 4. Dipole direction and amplitude from NVSS.

\begin{tabular}{lcccccc}
\hline \hline $\begin{array}{l}\text { Flux } \\
(\mathrm{mJy})\end{array}$ & $N$ & $\begin{array}{c}\text { RA } \\
\left({ }^{\circ}\right)\end{array}$ & $\begin{array}{c}\text { Dec } \\
\left({ }^{\circ}\right)\end{array}$ & $\begin{array}{c}d_{3 \mathrm{D}} \\
\left(10^{-2}\right)\end{array}$ & $k$ & $\begin{array}{c}d_{3 \mathrm{D}}^{\text {cor }} \\
\left(10^{-2}\right)\end{array}$ \\
\hline 50 & 91662 & $170 \pm 23$ & $-17 \pm 23$ & 2.78 & 1.38 & $2.0 \pm 0.8$ \\
40 & 115917 & $156 \pm 26$ & $-18 \pm 26$ & 2.23 & 1.29 & $1.7 \pm 0.8$ \\
35 & 133022 & $156 \pm 22$ & $-11 \pm 22$ & 2.46 & 1.32 & $1.9 \pm 0.7$ \\
30 & 155120 & $156 \pm 19$ & $-2 \pm 19$ & 2.63 & 1.35 & $1.9 \pm 0.7$ \\
25 & 185649 & $158 \pm 19$ & $-2 \pm 19$ & 2.38 & 1.34 & $1.8 \pm 0.6$ \\
20 & 229557 & $153 \pm 18$ & $2 \pm 18$ & 2.31 & 1.30 & $1.8 \pm 0.6$ \\
15 & 298289 & $149 \pm 18$ & $17 \pm 18$ & 2.02 & 1.28 & $1.6 \pm 0.5$ \\
\hline
\end{tabular}

Notes. The estimator (15) was used. Excluded are sources with $|\delta|>40^{\circ}$ and $|b|<10^{\circ}(\mathrm{J} 2000)$.

self-consistency indicates the absence of significant systematic errors. Unfortunately, we can not state the same for the declination results. One observes an significant increase in declination with respect to deceasing flux limits. This effect is very likely due to a relic of the NVSS survey procedure. Sources below $\delta=-10^{\circ}$ were measured by means of a different alignment of the Very Large Array. The source density is therefore smaller in this area. Therefore it is expected that the dipole measurement will show increasing values of declination at the fainter flux limits. This makes it hard to trust the declination results below a flux limit of $25 \mathrm{mJy}$ (note that there is no significant difference between $20 \mathrm{mJy}$ and $30 \mathrm{mJy}$ ).

The results of Table 4 can be compared to those in Table 2 . Number of sources and direction results are almost the same. Deviations could be explained by minor differences in the precise form of the mask. We used a different method for estimating uncertainties in the direction and amplitude measurement, than those used by Singal (2011). Since our method (described above) is more conservative, we obtain larger errorbars. All dipole amplitudes in this table are slightly below those from Table 2. In Singal (2011) a different method was used to obtain the dipole amplitude from the estimator (15), which can explain this discrepancy. In principle we can recover the results of Singal (2011) and confirm an unexpectedly high dipole amplitude.

\section{2. $2 D$ linear estimates}

A disadvantage of the NVSS catalogue is that the sampling depth changes at $\delta=-10^{\circ}$ (less sensitivity at lower declinations). This could lead to a directional bias of the NVSS data analysis and thus it is interesting to also use the two dimensional estimator presented in this work, as this effect cannot lead to a bias in this case. For the WENSS analysis, a three dimensional linear estimator cannot avoid directional bias.

\subsubsection{NVSS}

A major advantage of the estimator $\boldsymbol{R}_{2 \mathrm{D}}$ is that it does not require a north-south symmetry of the catalogue. Therefore, the declination limit of the NVSS catalogue is no problem.

As the estimator $\boldsymbol{R}_{2 \mathrm{D}}$ requires a point symmetry around the north pole, we cannot remove the Galactic plane only. For each removed point we also need to subtract the point which is $180^{\circ}$ away. When we do so, a second plane occurs, which we call the counter Galactic plane (CG). A HEALPix ${ }^{1}$ map of the remaining NVSS sources can be seen in Fig. 7. The colour of the pixels encodes the number of sources per pixel.

1 http://healpix.jpl.nasa.gov

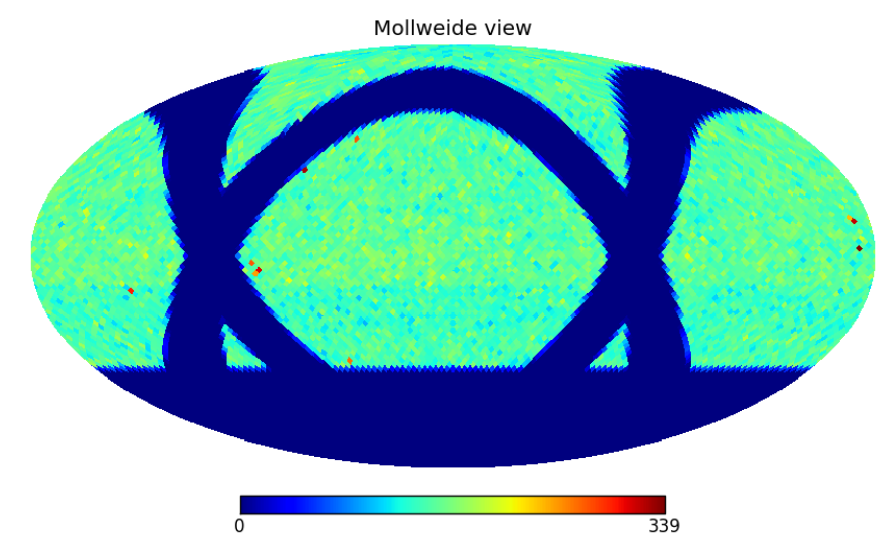

Fig. 7. Map of the number counts in HEALPix pixels from NVSS. The pixel size corresponds to $N_{\text {side }}=32$. Shown are equatorial coordinates at epoch J2000. The NVSS contains data at $\delta>-40^{\circ}$ and the Galactic plane and a "counter galaxy" are masked (CG mask) in order to avoid Galactic contamination and to restore point symmetry with respect to the zenith.

Table 5. Dipole right ascension and amplitude $d \sin \theta_{\mathrm{d}}$ from NVSS.

\begin{tabular}{lccccc}
\hline \hline $\begin{array}{l}\text { Flux } \\
(\mathrm{mJy})\end{array}$ & $N$ & $\begin{array}{c}\text { RA } \\
\left({ }^{\circ}\right)\end{array}$ & $\begin{array}{c}d_{2 \mathrm{D}} \\
\left(10^{-2}\right)\end{array}$ & $k$ & $\begin{array}{c}d_{2 \mathrm{D}}^{\text {cor }} \\
\left(10^{-2}\right)\end{array}$ \\
\hline 50 & 96337 & $171 \pm 19$ & 2.63 & 1.42 & $1.9 \pm 0.7$ \\
40 & 121831 & $146 \pm 20$ & 2.28 & 1.29 & $1.8 \pm 0.7$ \\
35 & 139851 & $152 \pm 17$ & 2.49 & 1.28 & $1.9 \pm 0.6$ \\
30 & 163208 & $153 \pm 15$ & 2.55 & 1.28 & $2.0 \pm 0.6$ \\
25 & 195245 & $155 \pm 14$ & 2.45 & 1.29 & $1.9 \pm 0.5$ \\
20 & 241399 & $150 \pm 14$ & 2.25 & 1.26 & $1.8 \pm 0.5$ \\
15 & 313724 & $148 \pm 15$ & 1.86 & 1.21 & $1.5 \pm 0.4$ \\
10 & 447459 & $133 \pm 14$ & 1.67 & 1.10 & $1.5 \pm 0.4$ \\
\hline
\end{tabular}

Notes. The 2D estimator (31) is used and all sources with $\delta>-40^{\circ}$, except the Galactic and counter Galactic planes (CG mask), are included.

An alternative would be the mask used by Singal (2011). That mask is a combination of two great cycles and would therefore also work for $\boldsymbol{R}_{2 \mathrm{D}}$. However, it turns out that the mask with the CG removes fewer sources. Thus we decided to use the $\mathrm{CG}$ mask.

The next step is to evaluate the masking correction $k$ of the $\mathrm{CG}$ mask. As this factor depends on the right ascension and on the declination of the dipole anisotropy, we need some additional information. From $\boldsymbol{R}_{2 \mathrm{D}}$ we estimate the right ascension. As the declination cannot be evaluated with $\boldsymbol{R}_{2 \mathrm{D}}$, we use the declination as provided by the three dimensional estimator in order to determine $k$. These values are certainly not exact, since a different mask is used now. The influence of a small change in dipole declination on the evaluated factor $k$ is discussed in Sect. 7.2.2.

We reduce the dipole amplitude of the 2D estimator by the masking factor $k$ and obtain the masking corrected amplitude $d_{2 \mathrm{D}}^{\text {cor }}$. The results of this procedure for the NVSS catalogue and different flux limits can be found in Table 5.

Obtained right ascensions and amplitudes are stable with respect to different flux limits. For all flux limits $\geq 15 \mathrm{mJy}$, the estimated right ascension of the radio dipole is in agreement with the CMB prediction of RA $=168^{\circ}$. Only when we include sources as faint as $10 \mathrm{mJy}$, we find a $3 \sigma$ deviation. However, we know that the catalogue is incomplete at its faint end.

The masking corrected dipole amplitudes $d_{2 \mathrm{D}}^{\text {cor }}$ are significantly above the $\mathrm{CMB}$ prediction. To some extent this is 


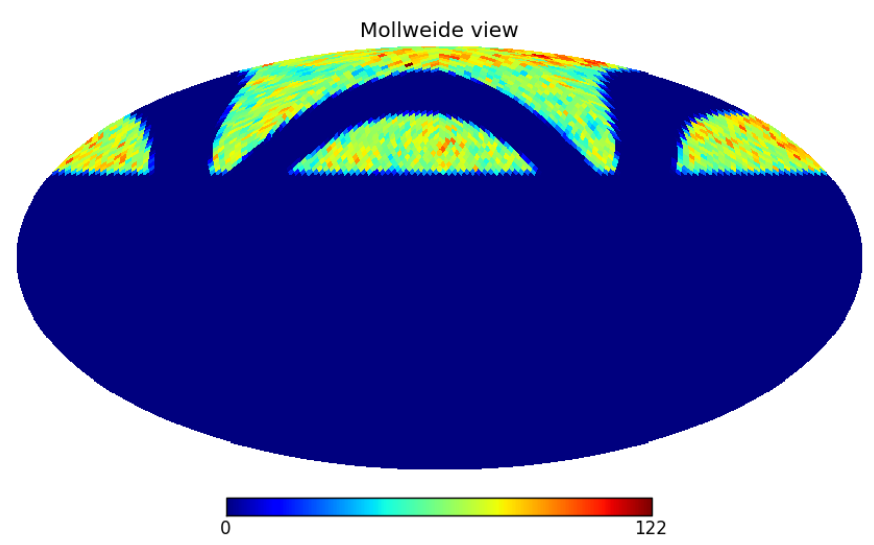

Fig. 8. Map of the number counts in HEALPix pixels from WENSS. The pixel size corresponds to $N_{\text {side }}=32$. Shown are equatorial coordinates at epoch B1950. The WENSS contains data at $\delta>30^{\circ}$ and the Galactic plane and a "counter galaxy" are masked (CG mask) in order to avoid Galactic contamination and to restore point symmetry with respect to the zenith.

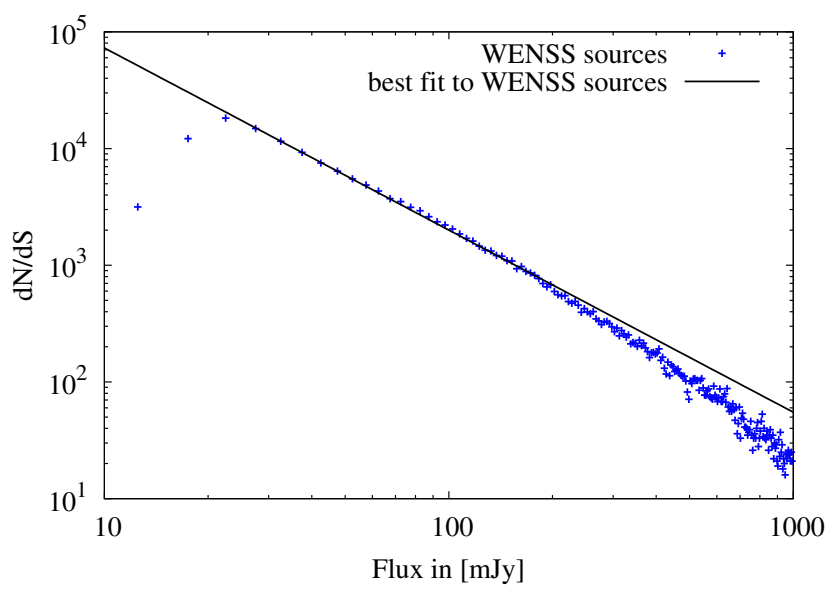

Fig. 9. Differential number counts of the WENSS catalogue, $S_{\min }=$ $5 \mathrm{mJy}$, best fit values for $f(s)=a \cdot s^{-\tilde{x}}$ with $25 \mathrm{mJy}<S<1000 \mathrm{mJy}$ are $a=2.6 \times 10^{6}$ and $\tilde{x}=1.6$.

expected due to the discussed amplitude bias. A detailed discussion is presented in the next section.

\subsubsection{WENSS}

We finally present the first estimation of the radio dipole from the WENSS catalogue. We cannot use the three dimensional linear estimators on this catalogue, since it only contains sources with Dec $>28^{\circ}$. The two dimensional estimator on the other hand can be used here. Again, we need to remove the Galactic plane and the counter Galactic plane to reestablish a symmetry around the north pole. The remaining WENSS catalogue is shown in Fig. 8.

To choose the best flux limit, we analyse the differential number counts between $10 \mathrm{mJy}$ and $1000 \mathrm{mJy}$. Figure 9 shows that the WENSS catalogue seems to be incomplete below 25 mJy. In Rengelink et al. (1997) the completeness of the WENSS catalogue is claimed to hold only above a limit of 30 mJy. From Fig. 9 we infer that a simple power law also applies to the source counts between 25 and $30 \mathrm{mJy}$ and thus we include sources down to a flux limit of $25 \mathrm{mJy}$ in our analysis.
Table 6. Masking correction $k$ for WENSS with CG mask and a dipole with RA $=120^{\circ}$.

\begin{tabular}{lccccccc}
\hline \hline Dec & $45^{\circ}$ & $30^{\circ}$ & $5^{\circ}$ & $0^{\circ}$ & $-5^{\circ}$ & $-30^{\circ}$ & $-45^{\circ}$ \\
\hline$k$ & 0.50 & 0.48 & 0.45 & 0.44 & 0.44 & 0.47 & 0.51 \\
\hline
\end{tabular}

Table 7. Dipole estimate from WENSS based on $2 \mathrm{D}$ estimator using peak flux values for all sources with $\delta>30^{\circ}$, except those in the Galactic and counter Galactic planes (CG mask).

\begin{tabular}{lccccc}
\hline \hline $\begin{array}{l}\text { Flux } \\
(\mathrm{mJy})\end{array}$ & $N$ & $\begin{array}{c}\text { RA } \\
\left({ }^{\circ}\right)\end{array}$ & $\begin{array}{c}d_{2 \mathrm{D}} \\
\left(10^{-2}\right)\end{array}$ & $k$ & $\begin{array}{c}d_{2 \mathrm{D}}^{\text {cor }} \\
\left(10^{-2}\right)\end{array}$ \\
\hline 40 & 67052 & $124 \pm 51$ & 1.31 & 0.45 & $2.9 \pm 2.3$ \\
35 & 73653 & $123 \pm 46$ & 1.36 & 0.47 & $2.9 \pm 2.1$ \\
30 & 81863 & $122 \pm 48$ & 1.24 & 0.45 & $2.8 \pm 2.1$ \\
25 & 92600 & $117 \pm 40$ & 1.36 & 0.47 & $2.9 \pm 1.9$ \\
\hline
\end{tabular}

Notes. Our WENSS analysis uses positions at epoch B1950.

We cannot obtain information on the declination of the dipole from the WENSS catalogue by means of the two dimensional estimator applied in this work. This could in principle be a problem for the determination of the masking factor $k$. Therefore, we further investigated the effect of different dipole declinations. Assuming the WENSS symmetry and a right ascension of $120^{\circ}$ (close to the results given in Table 7), we calculated $k$ for 7 different values of declination, see Table 6 . For this mask, the dependence of $k$ on the right ascension of the dipole is relatively small, compared to shot noise uncertainties. We assume dec $=0^{\circ}$ for the determination of $k$, based on the expectation from the CMB dipole and the NVSS radio dipole estimates.

The results of the WENSS analysis are presented in Table 7. Although the WENSS catalogue covers only one fourth of the sky, we find that it can be used for the estimation of the radio dipole. A problem is the limited number of sources that are left after masking the galaxy and restoring the required symmetry of the catalogue. This leads to larger error bars, compared with the NVSS estimates. We can conclude that the observed dipole anisotropy in the WENSS catalogue is in agreement with the measurements from NVSS, which is a nontrivial statement, as we are probing radio sources at different frequencies (325 MHz vs. $1.4 \mathrm{GHz}$ ).

\section{Comparison of results}

We summarize the various results from the literature and this work in Table 8 . The results of this work are highlighted with bold faced letters. For comparison we focus on the flux limits of $25 \mathrm{mJy}$ and $15 \mathrm{mJy}$.

All estimated dipole directions, both from the NVSS and from WENSS are in good agreement with each other and with the direction from the CMB dipole, with the exception of the result from Gibelyou \& Huterer (2012). As explained in Sect. 5.2, their estimator shows a directional bias. We did not investigate any further, whether this bias invalidates their findings at a rather low flux limit. Our analysis based on the three dimensional estimator applied to NVSS and using the mask defined by Singal $(2011)$ gives $(\mathrm{RA}, \mathrm{Dec})=\left(158^{\circ} \pm 19^{\circ},-2^{\circ} \pm 19^{\circ}\right)$.

For the amplitude of the radio dipole, the situation is more contrived. Here we focussed on the study of linear estimators and showed that all linear estimators under investigation returned a biased estimate of the amplitude. The amplitude estimators of Blake \& Wall (2002) and Gibelyou \& Huterer (2012) are unbiased, but the latter one uses a biased direction estimate as an 

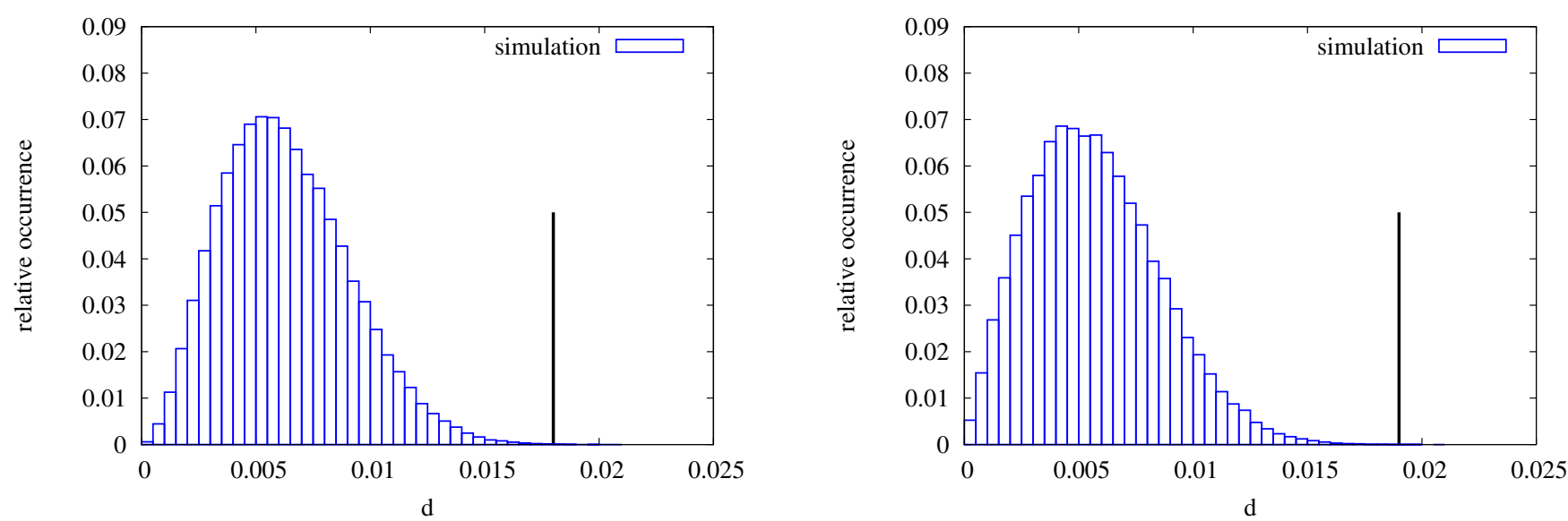

Fig. 10. Histogram of dipole amplitudes for 100,000 simulations of the three dimensional (left) and two dimensional (right) estimator, assuming the CMB expectation and a slope of $x=1.1$, with 185649 (left) and 195245 (right) sources per simulation and appropriate masks. The black vertical lines are the NVSS results of this work.

Table 8. Comparison of results.

\begin{tabular}{lccccc}
\hline \hline Source & $\begin{array}{c}\text { Flux }> \\
(\mathrm{mJy})\end{array}$ & $N$ & $\begin{array}{c}\text { RA } \\
\left({ }^{\circ}\right)\end{array}$ & $\begin{array}{c}\text { Dec } \\
\left({ }^{\circ}\right)\end{array}$ & $\begin{array}{c}d \\
\left(10^{-2}\right)\end{array}$ \\
\hline NVSS & & & & & \\
\hline BW & 25 & 197998 & $158 \pm 30$ & $-4 \pm 34$ & $1.1 \pm 0.3$ \\
SIN & 25 & 185474 & $158 \pm 10$ & $-2 \pm 10$ & $1.8 \pm 0.4$ \\
SIF & 25 & 184237 & $159 \pm 10$ & $-7 \pm 9$ & $2.2 \pm 0.6$ \\
SIF* & 25 & 184237 & $159 \pm 10$ & $-7 \pm 9$ & $\mathbf{1 . 6} \pm \mathbf{0 . 5}$ \\
3DS & $\mathbf{2 5}$ & $\mathbf{1 8 5 6 4 9}$ & $\mathbf{1 5 8} \pm \mathbf{1 9}$ & $-\mathbf{2} \pm \mathbf{1 9}$ & $\mathbf{1 . 8} \pm \mathbf{0 . 6}$ \\
2DCG & $\mathbf{2 5}$ & $\mathbf{1 9 5 2 4 5}$ & $\mathbf{1 5 5} \pm \mathbf{1 4}$ & $\ldots$ & $\mathbf{1 . 9} \pm \mathbf{0 . 5}$ \\
GH & 15 & 211487 & $117 \pm 20$ & $6 \pm 14$ & $2.7 \pm 0.5$ \\
3DS & $\mathbf{1 5}$ & $\mathbf{2 9 8 2 8 9}$ & $\mathbf{1 4 9} \pm \mathbf{1 8}$ & $\mathbf{1 7} \pm \mathbf{1 8}$ & $\mathbf{1 . 6} \pm \mathbf{0 . 5}$ \\
2DCG & $\mathbf{1 5}$ & $\mathbf{3 1 3 7 2 4}$ & $\mathbf{1 4 8} \pm \mathbf{1 5}$ & $\ldots$ & $\mathbf{1 . 5} \pm \mathbf{0 . 5}$ \\
\hline WENSS & & & & & \\
\hline 2DCG & $\mathbf{2 5}$ & $\mathbf{9 2 6 0 0}$ & $\mathbf{1 1 7} \pm \mathbf{4 0}$ & $\ldots$ & $\mathbf{2 . 9} \pm \mathbf{1 . 9}$ \\
\hline expected & \multicolumn{7}{c}{16} & -7 & $0.48 \pm 0.04$ \\
\hline NVSS & $\ldots$ & $\ldots$ & 168 & -7 & $0.42 \pm 0.03$ \\
WENSS & $\ldots$ & $\ldots$ & 168 &
\end{tabular}

Notes. Radio dipole from NVSS: BW (Blake \& Wall 2002), SIN (Singal 2011) number counts, SIF (Singal 2011) flux weighted number counts, SIF* corrects SIF for slope (this work), 3DS three-dimensional estimator, mask adopted from Singal (2011) (this work), 2DCG twodimensional estimator, CG mask (this work), GH (Gibelyou \& Huterer 2012); Radio dipole from WENSS: 2DCG two-dimensional estimator, CG mask (this work). The expectations for a purely kinetic radio dipole are given at the bottom of the table.

input and is thus of limited interest. Besides bias, we identified another effect that reduces the dipole amplitude found by the flux estimator used in Singal (2011). We reduced the result of this estimator by a factor of 1.4 , due to the fact that the appropriate exponent of the differential number count is given by $\tilde{x}=x+1$ (see Sect. 6). With this correction, the result of the flux weighted estimator (denoted by SIF* in Table 8 ) is now in agreement with the result of Blake \& Wall (2002).

Our three dimensional estimate with the masking of Singal (2011) gives rise to $d=(1.8 \pm 0.6) \times 10^{-2}$. One should keep in mind that this is a biased result, thus one cannot naively compare it to the expected amplitude. To figure out if our result is consistent with the null hypothesis that the radio sky is statistically isotropic, modified by the kinetic effects of our proper motion (measured via the CMB dipole), we performed 100000 Monte Carlo simulations. The corresponding histogram is shown in
Fig. 10. We find that only 21 of those realizations contain a dipole higher than the measured one and thus we can exclude that the estimated radio dipole is just due to our proper motion and amplitude bias at $99.6 \% \mathrm{CL}$. This is actually very puzzling, as the direction of the radio dipole agrees with the direction of the CMB dipole within the measurement error.

We can redo this analysis with the null hypothesis that the radio dipole was accurately measured by Blake \& Wall (2002). This time we find that 3402 out of 100000 realisations are higher than our measured dipole. If we increase the implemented velocity towards the upper one sigma bound of the dipole amplitude from Blake \& Wall (2002), we observe every sixth simulation to be above our own measurement (16\%). Therefore our result is in agreement with Blake \& Wall (2002).

Before we turn to the discussion of potential explanations, let us inspect the dipole amplitudes from the two dimensional estimator. The dipole amplitudes estimated with the two dimensional estimator are also in agreement with the results of Singal (2011) and Blake \& Wall (2002). We find $d \sin \vartheta_{\mathrm{d}}=(1.9 \pm 0.5) \times$ $10^{-2}$ for the NVSS analysis and $d \sin \vartheta_{\mathrm{d}}=(2.9 \pm 1.9) \times 10^{-2}$ for WENSS, which translate into lower limits on $d$. Thus, the results from the WENSS catalogue are in agreement with the radio dipole found in the NVSS catalogue. This is encouraging as they are prepared at different instruments and probe different frequencies.

In Figs. 10 and 11 we present the corresponding results from 100000 Monte Carlo simulations for the geometries of the NVSS and WENSS two dimensional estimators. In both cases we find that the amplitude bias is not enough to explain the difference between the observed and the expected amplitude. In the case of NVSS the null hypothesis (isotropic sky plus proper motion dipole) is ruled out at $99.6 \%$ CL, while for the WENSS analysis it is inconsistent at $98.1 \%$ CL.

The two dimensional estimation (2DCG), using the NVSS catalogue, is also compared to simulations, assuming the results from Blake \& Wall (2002). We observe 1141 and 7024 out of 100000 simulations to give a dipole above our measurement for the assumptions of $d_{\text {true }}=1.1 \times 10^{-2}$ and $d_{\text {true }}=1.4 \times 10^{-2}$, respectively. Therefore, the result of our two dimensional estimator is not in contradiction to Blake \& Wall (2002).

\section{Conclusion}

We conclude that the task to measure the cosmic radio dipole remains relevant and we expect that interesting information on 


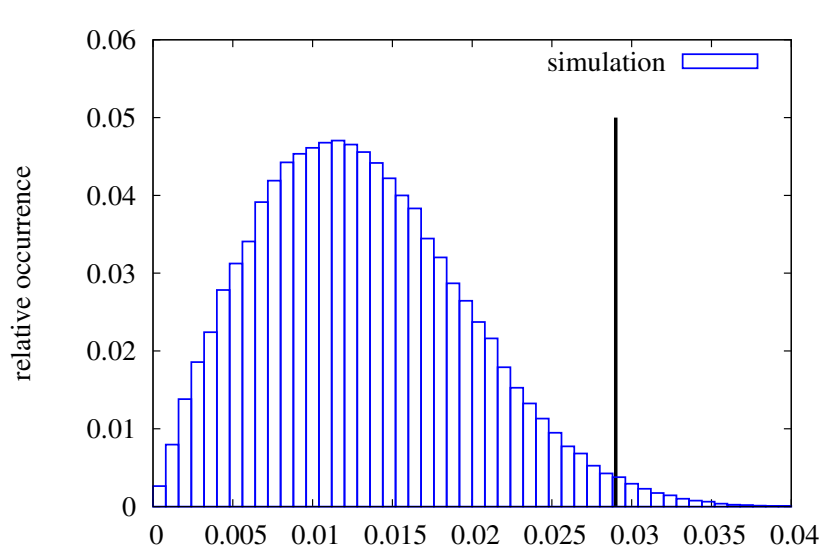

d

Fig. 11. Histogram of dipole amplitudes from 100000 simulations for the dimensional estimator, assuming the CMB expectation, a slope of $x=0.8,92,482$ sources per simulation and the CG masking form for the WENSS catalogue. The black vertical line is the WENSS result of this work

cosmology can be extracted from this measurement. All measurements so far point towards a higher radio dipole amplitude than expected, when we assume that the cosmic radio dipole is just due to our peculiar motion with respect to the rest frame defined by the CMB. This is quite puzzling, as the orientation of the radio dipole agrees with the orientation of the CMB dipole within measurement errors. This is the case for the NVSS and the WENSS analysis, two radio point source catalogues that cover $\sim 3 \pi$ and $\sim \pi$ of the sky, respectively. They contain information at different frequencies $(1.4 \mathrm{GHz}$ and $325 \mathrm{MHz})$ and have been put together by different instruments and thus provide a strong constraint on systematic issues.

Our detailed analysis of various linear dipole estimators (Crawford 2009; Singal 2011; Gibelyou \& Huterer 2012) for three dimensional estimates $\left(\alpha_{\mathrm{d}}, \delta_{\mathrm{d}}, d\right)$ and a new linear estimator for a two dimensional estimate $\left(\alpha_{\mathrm{d}}, d \cos \delta_{\mathrm{d}}\right)$ had to tackle several non-trivial issues. We investigated issues of directional bias, amplitude bias and masking. There is still room to optimize the masking of the galaxy. We did not look into quadratic estimators, as used by Blake \& Wall (2002). Our studies did not incorporate the uncertainties in point source positions, as we expect that they are subdominant (their magnitude is well below the effect of aberration, see e.g. Rengelink et al. 1997). The measurement error of the flux is also expected to be subdominant as we included sources with flux above $15 \mathrm{mJy}$ only. In the case of NVSS these are a factor 6 above the $5 \sigma$ point source detection limit, in case of WENSS it is a factor of 1.4. The number of point sources considered in our analysis is about 190000 for NVSS and 92000 for WENSS. Putting all those facts together, we consider the NVSS analysis to be more reliable. Nevertheless, the results of the WENSS analysis are fully consistent with our results from NVSS.

Our result (3DS) for the NVSS catalogue is (RA, Dec, $d)=$ $\left(154^{\circ} \pm 19^{\circ},-2^{\circ} \pm 19^{\circ},(1.8 \pm 0.6) \times 10^{-2}\right)$. Thus we conclude that the observed amplitude of the radio dipole exceeds the expected amplitude by about a factor of four. We could imagine that this might be due to the structure that causes our proper motion, which in a simple model of our Hubble patch would certainly be aligned with the direction of proper motion. However, all attempts to identify a "great attractor" by means of other observations (optical, infra-red, X-ray) have failed so far. Of all those probes, the radio surveys are definitely the deepest probe of the Universe, as the mean redshift of NVSS sources is estimated to be 1.2 by de Zotti et al. (2010) and 1.5 by Ho et al. (2008). To explain the observed excess radio dipole by contributions from local structure, we would need a density contrast of order 0.05 at scales extending to about $z \approx 0.3$, which does not seem plausible. Without a detailed study of the redshift distribution of the radio sources it is impossible to judge whether this finding is actually in agreement with the current standard model of cosmology.

An example of such a scenario is provided by Wiltshire et al. (2012). They claim that the spherically averaged Hubble law on $<100 / h$ Mpc scales is significantly closer to uniform in the Local Group frame as compared to the CMB frame and on this basis have suggested a non-kinematic contribution to the CMB dipole. In this case the CMB dipole could differ from the cosmic radio dipole.

Another reason for the large amplitude of the radio dipole could be that the linear estimators considered in this work do not assume the deviation from isotropy to be a pure dipole. Thus higher multipole moments also contribute to the measured amplitude.

It has been found from the analysis of the $\mathrm{CMB}$ that quadrupole, octopole and a few more low $\ell$-multipoles seem not to be orientated randomly on the sky, but show some unexpected alignments (Schwarz et al. 2004; Bennett et al. 2011; Copi et al. 2010) among themselves and with the CMB dipole direction. Thus it might not be surprising that also the dominant anisotropy direction of the radio sky lines up.

It is evident that it would be crucial to repeat this investigation with new and even deeper radio catalogues, which provide more sources. In the near future there will be three large sky surveys available (Raccanelli et al. 2012). A multi-wavelength study will be possible based on the Multifrequency Snapshot Sky Survery (MSSS) of the International LOFAR Telescope and with the LOFAR Tier-1 survey. The Australian Square Kilometre Array (SKA) Pathfinder (ASKAP) will produce the Evolutionary Map of the Universe (EMU) and the Westerbrok Synthesis Radio Telescope (WSRT) equipped with the Apertif system will compile the Westerbrok Observations of the Deep Apertif Northern sky survey (WODAN) catalogue.

The multi-wavelength surveys will also allow us to directly measure the spectral index $\alpha$, which has to be known to connect the measured amplitude to the kinetic dipole. A steepening of the spectral index for the lowest flux sources would increase the expected kinetic amplitude. HI surveys will have the advantage that they will also provide redshift information on top of positions and fluxes and we will be able to study the evolution of the radio dipole as a function of redshift out to redshifts of a few. Beyond that SKA will increase the number of sources in such a survey by orders of magnitude. All these surveys will reduce the random dipole contribution, improve on systematics, and allow us to settle the question: Is the radio dipole in agreement with the CMB dipole?

Acknowledgements. We thank David J. Bacon, Dragan Huterer, Matt J. Jarvis, Seshadri Nadathur, Huub Röttgering and Ashok K. Singal for fruitful comments and discussions and Anne-Sophie Balleier for help with Healpy and the generation of maps of the NVSS and WENSS number counts. The CAMB code, and the Healpy and HEALPix (Górski et al. 2005) packages have been used to estimate the concordance value of the CMB primordial dipole and to produce the NVSS and WENSS maps of the sky. We acknowledge financial support from Deutsche Forschungsgemeinschaft (DFG) under grants IRTG 881 'Quantum Fields and Strongly Interacting Matter' and RTG 1620 'Models of Gravity', as well as from the Friedrich Ebert Stiftung. 


\section{Appendix A: Monte Carlo simulations}

We used the pseudorandom number generator Mersenne Twister for all Monte Carlo simulations. One simulated source consists of two position coordinates and one flux value. The coordinates will be drawn from an uniform distribution, leading to an isotropic sky for a catalogue with many sources. To obtain a desired number count power law like (4) with a certain slope $x$, we calculate the flux $S$ using a random number $A$ (choosen between 0 and 1) by

$S_{\text {rest }}=S_{\mathrm{L}}(1-A)^{-x}$,

where $S_{\mathrm{L}}$ is a flux value $20 \%$ below the simulated flux limit. The simulation also creates sources, which only due to Doppler shifting are counted in the final catalogue, because this value of $S_{\mathrm{L}}$ lies below the simulated flux limit. Lowering $S_{\mathrm{L}}$ further increases computational time and is not necessary, as long as the simulated velocity $v$ is below $0.1 c$.

The two physical effects (Doppler shift and spherical aberration) will be implemented separately. In cooperating the Doppler effect is straightforward, since it only affects the flux values of each source depending on the angle $\theta$ between the source and the velocity direction, i.e.

$S_{\text {obs }}\left(v_{\text {obs }}\right)=S_{\text {rest }}\left(\frac{1+\frac{v}{c} \cos (\theta)}{\sqrt{1-\left(\frac{v}{c}\right)^{2}}}\right)^{1+\alpha}$.

In the simulation $\alpha$ is fixed to 0.75 for all sources. The velocity direction and amplitude can by chosen be the user.

To model the relativistic effect of stellar aberration one has to change the position of each radio source. The aberration formula is

$\tan \left(\theta^{\prime}\right)=\frac{\sin (\theta) \sqrt{1-\left(\frac{v}{c}\right)^{2}}}{\frac{v}{c}+\cos (\theta)}$,

where $\theta^{\prime}$ is the new angle between the velocity direction and the radio source. Forth, the position of a radio source is translated into Cartesian coordinates by assuming that it lies on a unit sphere. Then a straight line from this point $(\boldsymbol{P})$ to the velocity direction on the sphere $(\boldsymbol{V})$ is constructed depending on a parameter $t$

$\boldsymbol{r}(t)=\boldsymbol{P}(1-t)+\boldsymbol{V} t$.

On this line we choose a $t^{\prime}$ in such a way that $\boldsymbol{r}\left(t^{\prime}\right)$ points towards the new position. The value of $t^{\prime}$ can be determined by

$\boldsymbol{r}\left(t^{\prime}\right) \cdot \boldsymbol{V}=r^{\prime}\left(t^{\prime}\right) \cos \left(\theta^{\prime}\right)$

$\rightarrow t^{\prime}=\frac{r^{\prime}\left(t^{\prime}\right) \cos \left(\theta^{\prime}\right)-\cos (\theta)}{1-\cos (\theta)}$ with $r^{\prime}\left(t^{\prime}\right)=\sqrt{\boldsymbol{r}^{\prime 2}\left(t^{\prime}\right)}$. This equation is solved by

$t_{1}^{\prime}=\frac{\sin \left(\theta-\theta^{\prime}\right)}{\sin \left(\theta-\theta^{\prime}\right)+\sin \left(\theta^{\prime}\right)} \vee t_{2}^{\prime}=\frac{\sin \left(\theta+\theta^{\prime}\right)}{\sin \left(\theta+\theta^{\prime}\right)-\sin \left(\theta^{\prime}\right)}$.

We know that for $\theta=\theta^{\prime}$ the result of $t^{\prime}$ must always be 0 . Therefore the correct solution is $t^{\prime}=t_{1}^{\prime}$. Now one has to transform $\boldsymbol{r}\left(t^{\prime}\right)$ back into spherical coordinates in order to find the new position of the radio source. The new declination $\vartheta^{\prime}$ is then (the index $\mathrm{v}$ stands for the velocity direction):

$\vartheta^{\prime}=\arccos \left(\frac{1}{r^{\prime}}\left[\left(1-t^{\prime}\right) \cos (\vartheta)+t^{\prime} \cos \left(\vartheta_{\mathrm{v}}\right)\right]\right)$

and the new right ascension $\varphi^{\prime}$ :

$\varphi^{\prime}=\arcsin \left(\frac{\left(1-t^{\prime}\right) \sin (\vartheta) \sin (\varphi)+t^{\prime} \sin \left(\vartheta_{\mathrm{v}}\right) \sin \left(\alpha_{\mathrm{v}}\right)}{r^{\prime} \sin \left(\vartheta^{\prime}\right)}\right)$.

This way one obtains a simulated sky, including the effect of the observers movement. Now one can feed the different estimators with those sky simulations and obtain the resulting dipole vectors.

\section{References}

Baleisis, A., Lahav, O., Loan, A. J., \& Wall, J. V. 1998, MNRAS, 297, 545 Bennett, C. L., Hill, R. S., Hinshaw, G., et al. 2011, ApJS, 192, 17 Blake, C., \& Wall, J. 2002, Nature, 416, 150

Condon, J. J., Cotton, W. D., Greisen, E. W., et al. 2002, VizieR Online Data Catalog, VIII/065

Copi, C. J., Huterer, D., Schwarz, D. J., \& Starkman, G. D. 2010, Adv. Astron., 2010, id. 847541

Crawford, F. 2009, ApJ, 692, 887

de Zotti, G., Massardi, M., Negrello, M., \& Wall, J. 2010, A\&A Rev., 18, 1

Ellis, G. F. R., \& Baldwin, J. E. 1984, MNRAS, 206, 377

Fixsen, D., \& Mather, J. 2002, ApJ, 581, 817

Francis, C., \& Peacock, J. 2010, MNRAS, 406, 14

Garn, T., Green, D. A., Riley, J. M., \& Alexander, P. 2008, MNRAS, 383, 75

Gibelyou, C., \& Huterer, D. 2012, MNRAS, 427, 1994

Górski, K. M., Hivon, E., Banday, A. J., et al. 2005, ApJ, 622, 759

Hinshaw, G., Weiland, J. L., Hill, R. S., et al. 2009, ApJS, 180, 225

Hirata, C. M. 2009, , J. Cosmol. Astropart. Phys., 9, 11

Ho, S., Hirata, C., Padmanabhan, N., Seljak, U., \& Bahcall, N. 2008, Phys. Rev. D, 78, 043519

Raccanelli, A., Zhao, G.-B., Bacon, D. J., et al. 2012, MNRAS, 424, 801

Rakic, A., Rasanen, S., \& Schwarz, D. J. 2006, MNRAS, 369, L27

Rengelink, R. B., Tang, Y., de Bruyn, A. G., et al. 1997, A\&AS, 124, 259

Rubart, M. 2012, Master Thesis, Universität Bielefeld

Schwarz, D. J., Starkman, G. D., Huterer, D., \& Copi, C. J. 2004, Phys. Rev. Lett., 93, 221301

Singal, A. K. 2011, ApJ, 742, L23

Stewart, J. M., \& Sciama, D. W. 1967, Nature, 216, 748

Wiltshire, D. L., Smale, P. R., Mattsson, T., \& Watkins, R. 2012

[arXiv: 1201.5371] 